Supporting information:

\title{
Total Synthesis of Herboxidiene/GEX 1A
}

\author{
Yun Zhang and James S. Panek* \\ Department of Chemistry and Center for Chemical Methodology and Library \\ Development, Metcalf Center for Science and Engineering \\ Boston University, Boston, Massachusetts 02215
}

\section{General Information.}

All reactions were carried out in oven dried glassware under an argon atmosphere employing standard techniques in handling air-sensitive materials. All solvents were reagent grade. Dichloromethane (DCM) was freshly distilled from calcium hydride, tetrahedrofuran (THF) was freshly distilled from sodium/benzophenone under argon immediately prior to use. Trimethylsilyltrifluoromethanesulfonate (TMSOTf) was freshly distilled before use. All other reagents were used as supplied. Unless otherwise noted, reactions were magnetically stirred and monitored by thin layer chromatography with Sorbent technologies $0.20 \mathrm{~mm}$ silica gel 60 plates. Flash chromatography were performed with silica gel 60 (particle size $0.032-0.063 \mathrm{~mm}$ ) supplied by Sorbent Technologies. Yields refer to chromatographically and spectroscopically pure compounds, unless otherwise noted. ${ }^{1} \mathrm{H}$ NMR spectra were recorded using an internal deuterium lock at ambient temperature on a Varian $400 \mathrm{MHz}$ spectrometer. An internal reference of $\delta_{\mathrm{H}}$ 7.24 was used for $\mathrm{CDCl}_{3}$. Data are presented as follows: chemical shift (in ppm on the $\delta$ scale relatively to $\delta_{\mathrm{TMS}}=0$ ), multiplicity $(\mathrm{s}=$ singlet, $\mathrm{d}=$ doublet, $\mathrm{t}=$ triplet, $\mathrm{q}=$ quartet, quint. = quintuplet, $\mathrm{m}=$ mutiplet, $\mathrm{br}=$ broad, $\mathrm{ABq}=\mathrm{AB}$ quartet, $\mathrm{dd}=$ doublet of doublet, $\mathrm{dt}=$ doublet of triplet, $\mathrm{dq}=$ doublet of quartet $)$, coupling constant $(\mathrm{J} / \mathrm{Hz})$ and integration. Resonances that are either partially or fully obscured are denoted obscured (obsc). ${ }^{13} \mathrm{C}$ NMR spectra were recorded on a Varian $400 \mathrm{MHz}$ spectrometer. An internal reference of $\delta_{\mathrm{C}} 77.0$ was used for $\mathrm{CDCl}_{3}$. Infrared spectra were recorded on a Nexus 670 FTIR spectraphotometer. Optical rotations were recorded on an Autopol III digital polarimeter at $589 \mathrm{~nm}$ and reported as follows: $[\alpha]_{\mathrm{D}}{ }^{20}$, concentration $(c$ in $\mathrm{g} / 100 \mathrm{~mL})$ and solvent. High resolution mass spectra were obtained on a Finnagan MAT-90 spectrometer in the Boston University Mass Spectrometer Laboratory. 


\section{Experimental procedures}

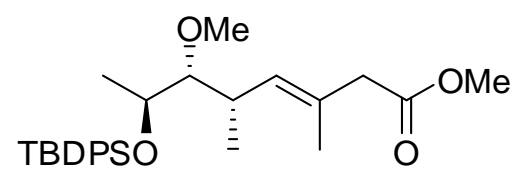

$(3 E, 5 S, 6 S, 7 S)$ 7-(tert-Butyl-diphenyl-silanyloxy)-6methoxy-3,5-dimethyl-oct-3-enoic acid methyl ester (11). A solution of $(S)$-crotylsilane $12(1.67 \mathrm{~g}, 6.0 \mathrm{mmol})$ and dimethyl acetal $\mathbf{1 3}^{1}(2.58 \mathrm{~g}, 7.2 \mathrm{mmol})$ in DCM (12 $\mathrm{mL}, 0.5 \mathrm{M})$ was cooled to $-78^{\circ} \mathrm{C}$, and TMSOTf $(3.48 \mathrm{~mL}, 18.0 \mathrm{mmol})$ was charged in. The reaction mixture was slowly warmed up to $-50^{\circ} \mathrm{C}$ and stirred for $24 \mathrm{~h}$ before being quenched with saturated $\mathrm{NaHCO}_{3}$ solution $(30 \mathrm{~mL})$ and the aqueous layer was extracted with DCM (3 $\times 20 \mathrm{~mL})$. The combined organic layers were washed with brine, dried over anhydrous $\mathrm{MgSO}_{4}$ and concentrated under pressure. Column chromatography on silica gel (2-5\% EtOAc/hexane eluant) gave $1.696 \mathrm{~g}$ product as a pale yellow oil $(62 \%) .{ }^{1} \mathrm{H}$ NMR $\left(400 \mathrm{MHz}, \mathrm{CDCl}_{3}\right) \delta 7.67-7.65(\mathrm{~m}, 4 \mathrm{H}), 7.43-7.33(\mathrm{~m}, 6 \mathrm{H}), 4.68(\mathrm{~d}, \mathrm{~J}=10.4 \mathrm{~Hz}$, $1 \mathrm{H}), 3.82(\mathrm{dq}, \mathrm{J}=2.8,6.0 \mathrm{~Hz}, 1 \mathrm{H}), 3.57(\mathrm{~s}, 3 \mathrm{H}), 3.53(\mathrm{~s}, 3 \mathrm{H}), 2.88(\mathrm{dd}, \mathrm{J}=2.4,8.0 \mathrm{~Hz}$, $1 \mathrm{H}), 2.77(\mathrm{~s}, 2 \mathrm{H}), 2.33(\mathrm{~m}, 1 \mathrm{H}), 1.52(\mathrm{~s}, 3 \mathrm{H}), 1.04(\mathrm{~s}, 9 \mathrm{H}), 1.01(\mathrm{~d}, \mathrm{~J}=6.4 \mathrm{~Hz}, 3 \mathrm{H}), 0.86$ $(\mathrm{d}, \mathrm{J}=6.4 \mathrm{~Hz}, 3 \mathrm{H}) ;{ }^{13} \mathrm{C} \mathrm{NMR}\left(400 \mathrm{MHz}, \mathrm{CDCl}_{3}\right) \delta 172.1,136.0,134.5,132.3,129.6$, 127.5, 89.7, 71.3, 61.2, 51.5, 44.8, 35.3, 27.0, 19.2, 17.1, 16.8, 16.3; IR (Neat) $v_{\max } 3000$, 2930, 2857, 1739, 1428, 1384, 1259, 1156, 1101, 1044, 933, 822, 772, 740, 702; HRMS $\left(\mathrm{Cl} / \mathrm{NH}_{3}\right) \mathrm{m} / \mathrm{z}$ calcd for $\mathrm{C}_{28} \mathrm{H}_{40} \mathrm{O}_{4} \mathrm{Si}[\mathrm{M}+\mathrm{Na}]^{+} 491.2594$ found $491.2599 ;[\alpha]^{20}{ }_{\mathrm{D}}=+21.0^{\circ}(c$ $\left.1.1, \mathrm{CHCl}_{3}\right)$.<smiles></smiles>

$(6 S, 7 R, 8 S, E)-8$-(tert-butyldiphenylsilyloxy)-1diazo-7-methoxy-4,6-dimethylnon-4-en-2-one (14). A solution of methyl ester $11(1.69 \mathrm{~g}, 3.61 \mathrm{mmol})$ in THF-MeOH- $\mathrm{H}_{2} \mathrm{O}(3: 1: 1)(14.4 \mathrm{~mL}, 0.25 \mathrm{M})$ was added $\mathrm{LiOH}$ (303mg, $7.22 \mathrm{mmol}$ ) at rt. The heterogeneous reaction mixture was stirred at $\mathrm{rt}$ for another $3 \mathrm{~h}$ before being diluted with diethyl ether $10 \mathrm{~mL}$ and careful acidification with $1 \mathrm{~N} \mathrm{HCl}$ solution till $\mathrm{PH}=3.0$. The aqueous layer was extracted with diethyl ether $(3 \times 10 \mathrm{~mL})$. The combined organic layers were washed with brine, dried over anhydrous $\mathrm{MgSO}_{4}$ and concentrated under pressure to give the crude reaction product as viscous pale yellow oil. No further purification needed.

A solution of the crude acid in DCM $(18.8 \mathrm{~mL}, 0.2 \mathrm{M})$ was treated with the freshly distilled oxallyl chloride $(394 \mu \mathrm{l}, 4.52 \mathrm{mmol})$ at $\mathrm{rt}$ followed by slow addition of anhydrous $\operatorname{DMF}(30 \mu \mathrm{L}, 0.38 \mathrm{mmol})$. The reaction mixture was stirred at $\mathrm{rt}$ for another $15 \mathrm{~min}$ before being concentrated under pressure to give a yellow residue. A solution of the crude acid chloride in diethyl ether ( $4 \mathrm{~mL}, 1 \mathrm{M}$ ) was added into a freshly prepared $\mathrm{CH}_{2} \mathrm{~N}_{2}$ (ca.20 eq.) diethyl ether solution at $0{ }^{\circ} \mathrm{C}$, stirring was maintained at that Temp for 30 min before being quenched with $\mathrm{DI}_{2} \mathrm{O} 20 \mathrm{~mL}$. The reaction mixture was allowed to warm to $\mathrm{rt}$ and stirred for another $20 \mathrm{~min}$ until no gas evolution observed. The aqueous layer was extracted with diethyl ether $(3 \times 20) \mathrm{mL}$. The combined organic layers were washed with brine, dried over anhydrous $\mathrm{MgSO}_{4}$ and concentrated under pressure. Column chromatography on silica gel (10\% EtOAc/hexane eluant) gave $1.68 \mathrm{~g}$ product as bright

\footnotetext{
${ }^{1}$ Details of prepration of the corresponding $(\alpha)$-siloxy aldehyde, see: Smith, N. D.; Kocieński, P. J.; Street S. D. A. Synthesis 1996, 652. The aldehyde was then converted to dimethylacetal 13 using TMSOMe and catalytic amounts of TMSOTf in DCM at $-50{ }^{\circ} \mathrm{C}$ stirring for $12 \mathrm{~h}$ followed by conventional work-up. This material was used without any further purification.
} 
yellow oil (97\% in 2 steps). ${ }^{1} \mathrm{H}$ NMR $\left(400 \mathrm{MHz}, \mathrm{CDCl}_{3}\right) \delta 7.68-7.64(\mathrm{~m}, 4 \mathrm{H}), 7.45-$ $7.32(\mathrm{~m}, 6 \mathrm{H}), 4.77(\mathrm{~s}, 1 \mathrm{H}), 4.72(\mathrm{~d}, \mathrm{~J}=10 \mathrm{~Hz}, 1 \mathrm{H}), 3.82(\mathrm{dq}, \mathrm{J}=2.0,6.0 \mathrm{~Hz}, 1 \mathrm{H}), 3.61$ (s, $3 \mathrm{H}), 2.95(\mathrm{~d}, \mathrm{~J}=7.2 \mathrm{~Hz}, 1 \mathrm{H}), 2.73$ (brs, 2H), $2.33-2.26(\mathrm{~m}, 1 \mathrm{H}), 1.47(\mathrm{~s}, 3 \mathrm{H}), 1.04(\mathrm{~s}$, $9 \mathrm{H}), 1.00(\mathrm{~d}, \mathrm{~J}=6.4 \mathrm{~Hz}, 3 \mathrm{H}), 0.89(\mathrm{~d}, \mathrm{~J}=6.4 \mathrm{~Hz}, 3 \mathrm{H}) ;{ }^{13} \mathrm{C} \mathrm{NMR}\left(400 \mathrm{MHz}, \mathrm{CDCl}_{3}\right) \delta$ 193.3, 136.0, 135.8, 133.1 129.7, 129.6, 127.5, 89.5, 71.5, 61.2, 52.5, 35.6, 26.9, 19.1, 17.2, 16.8, 16.1; IR (Neat) $v_{\max } 3071,2959,2932,2858,2103,1643,1472,1347,1102$, 1045, 933, 822; HRMS $\left(\mathrm{Cl} / \mathrm{NH}_{3}\right) \mathrm{m} / z$ calcd for $\mathrm{C}_{28} \mathrm{H}_{38} \mathrm{~N}_{2} \mathrm{O}_{3} \mathrm{Si}[\mathrm{M}+\mathrm{Na}]^{+} 501.2561$ found 501.2549; $[\alpha]^{20}{ }_{\mathrm{D}}=+46.3^{\circ}\left(c 1.8, \mathrm{CHCl}_{3}\right)$.<smiles>CO[13C](=O)O[C@@H](C)[C@@H](C)[C@H](C)/C=C(\C)CCC(=O)N(C)[C@@H](C)[C@H](O)c1ccccc1</smiles>

(6S, $\quad 7 R, \quad 8 S, \quad E)-8$-(tertbutyldiphenylsilyloxy)-N-((1S,2S)-1hydroxy-1-phenylpropan-2-yl)-7-methoxyN,4,6-trimethylnon-4-enamide (15). A diazoketone $14(1.68 \mathrm{~g}, 3.51 \mathrm{mmol})$ solution in DCM (70 mL, $0.05 \mathrm{M})$ was added silver benzoate $(804 \mathrm{mg}, 3.51 \mathrm{mmol})$ followed by addition of $(1 S, 2 S)-(+)$-pseudoephedrine $(1.16 \mathrm{~g}, 7.025 \mathrm{mmol})$. The brown reaction mixture was stirred at $\mathrm{rt}$ for $5 \mathrm{~min}$ before being quenched with $\mathrm{H}_{2} \mathrm{O} 20 \mathrm{~mL}$. The aqueous layer was extracted with DCM (3X). The combined organic layers were washed with brine, dried over anhydrous $\mathrm{MgSO}_{4}$ and concentrated under pressure to give a yellow residue. Column chromatography on silica gel (50\% EtOAc/hexane eluant) gave 1.73g product as white solid $(80 \%)$. ${ }^{1} \mathrm{H}$ NMR $(2: 1$ rotamer ratio, asterisk denotes minor rotamer peaks, $\left.400 \mathrm{MHz}, \mathrm{CDCl}_{3}\right) \delta 7.64-7.61(\mathrm{~m}, 4 \mathrm{H}) 7.42-7.25(\mathrm{~m}, 11 \mathrm{H}), 4.69 *(\mathrm{~d}, \mathrm{~J}=12.8$ $\mathrm{Hz}, 1 \mathrm{H}), 4.66(\mathrm{~d}, \mathrm{~J}=10.4 \mathrm{~Hz}, 1 \mathrm{H}), 4.57(\mathrm{t}, \mathrm{J}=7.2 \mathrm{~Hz}, 1 \mathrm{H}), 4.55^{*}(\mathrm{t}, \mathrm{J}=10.4 \mathrm{~Hz}, 1 \mathrm{H})$, 4.50 - 4.38 (m, 1H), 4.2 (brs, 1H), 3.93* (quint, $\mathrm{J}=8.4 \mathrm{~Hz}, 1 \mathrm{H}), 3.84$ (dq, J = 2.4, $6.0 \mathrm{~Hz}$, $1 \mathrm{H}), 3.59(\mathrm{~s}, 3 \mathrm{H}), 3.57 *(\mathrm{~s}, 3 \mathrm{H}), 2.92(\mathrm{dd}, \mathrm{J}=2.4,8.4 \mathrm{~Hz}, 1 \mathrm{H}), 2.90^{*}(\mathrm{~s}, 3 \mathrm{H}), 2.72(\mathrm{~s}, 3 \mathrm{H})$, $2.30-2.24(\mathrm{~m}, 1 \mathrm{H}), 2.19-2.01(\mathrm{~m}, 2 \mathrm{H}), 1.61(\mathrm{~s}, \mathrm{OH}), 1.47 *(\mathrm{~s}, 3 \mathrm{H}), 1.44(\mathrm{~s}, 3 \mathrm{H}), 1.09(\mathrm{~d}$, $\mathrm{J}=6.8 \mathrm{~Hz}, 3 \mathrm{H}), 1.04(\mathrm{~s}, 9 \mathrm{H}), 0.97(\mathrm{~d}, \mathrm{~J}=6.4 \mathrm{~Hz}, 3 \mathrm{H}), 0.86(\mathrm{~d}, \mathrm{~J}=6.4 \mathrm{~Hz}, 3 \mathrm{H}) ;{ }^{13} \mathrm{C} \mathrm{NMR}$ $\left(2: 1\right.$ rotamer ratio, sterisk denotes minor rotamer peaks, $\left.400 \mathrm{MHz}, \mathrm{CDCl}_{3}\right) \delta 175.1$, $173.8^{*}, 142.4,140.5^{*}, 135.9,134.5,133.9,133.1,129.7,129.5^{*}, 128.7^{*}, 128.4,127.7$, $127.5^{*}, 126.8^{*}, 126.4,89.8,76.6,71.4,61.1,58.4,58.3^{*}, 35.2,34.6,33.2,32.5^{*}, 27.0$, $19.2,17.3,17.0,16.2,15.4^{*}, 14.5$; IR (Neat) $v_{\max } 2952,2927,2867,1735,1453,1434$, 1334, 1258, 1190, 1155, 1069, 1028, 1004, 736, 712, 698; $\mathrm{HRMS}\left(\mathrm{Cl} / \mathrm{NH}_{3}\right) \mathrm{m} / z$ calcd for $\mathrm{C}_{38} \mathrm{H}_{53} \mathrm{NO}_{4} \mathrm{Si}[\mathrm{M}+\mathrm{H}]^{+} 616.3822$ found 616.3866; $[\alpha]^{20} \mathrm{D}=+46.3^{\circ}\left(c 1.7, \mathrm{CHCl}_{3}\right)$.<smiles></smiles>

$(2 S, \quad 6 S, \quad 7 R, \quad 8 S, \quad E)-8$-(tertbutyldiphenylsilyloxy)-N-((1S, $\quad 2 S)-1-$ hydroxy-1-phenylpropan-2-yl)-7methoxy-N,2,4,6-tetramethylnon-4-

enamide (16). A schlenk flask was charged with lithium chloride (331 $\mathrm{mg}, 7.8 \mathrm{mmol}$ ) and was flammable dried under high vacuum. Freshly distilled diisopropylamine $(439 \mu \mathrm{L}, 3.13 \mathrm{mmol})$ and anhydrous THF $(0.83 \mathrm{M}$, $1.57 \mathrm{~mL})$ were added in. $\mathrm{n}-\mathrm{BuLi}(2.50 \mathrm{M}$ in hexanes, $1.17 \mathrm{~mL}, 2.9 \mathrm{mmol})$ was charged in at $-78^{\circ} \mathrm{C}$. The mixture was warmed up to $0{ }^{\circ} \mathrm{C}$ and was stirred at that temperature for $10 \mathrm{~min}$. The mixture was cooled back to $-78{ }^{\circ} \mathrm{C}$ and a solution of amide $15(803 \mathrm{mg}, 1.3$ $\mathrm{mmol})$ in dry THF $(0.19 \mathrm{M}, 7 \mathrm{~mL})$ was added very slowly at that Temp. The yellow reaction mixture was stirred at $-78{ }^{\circ} \mathrm{C}$ for $1 \mathrm{~h}$ before being warmed up to $0{ }^{\circ} \mathrm{C}$ and was 
stirred at that Temp for another $45 \mathrm{~min}$. MeI $(405 \mu \mathrm{L}, 6.5 \mathrm{mmol}$, passed though a short column of $\mathrm{Al}_{2} \mathrm{O}_{3}$ before using) was added slowly into the reaction mixture and stirring was continued at that Temp for $30 \mathrm{~min}$ before being quenched at $0{ }^{\circ} \mathrm{C}$ with saturated $\mathrm{NH}_{4} \mathrm{Cl}$ solution. The aqueous layer was extracted with diethyl ether (3X). The combined organic layers were washed with brine, dried over anhydrous $\mathrm{MgSO}_{4}$ and concentrated under pressure to give a yellow sticky solid $(787 \mathrm{mg}, 96.1 \%$ yield $) .{ }^{1} \mathrm{H}$ NMR $(2: 1$ rotamer ratio, asterisk denotes minor rotamer peaks, $\left.400 \mathrm{MHz}, \mathrm{CDCl}_{3}\right) \delta 7.67-7.64(\mathrm{~m}, 4 \mathrm{H})$, $7.42-7.31(\mathrm{~m}, 11 \mathrm{H}), 4.71(\mathrm{~d}, \mathrm{~J}=10.4 \mathrm{~Hz}, 1 \mathrm{H}), 4.60(\mathrm{t}, \mathrm{J}=7.2 \mathrm{~Hz}, 1 \mathrm{H}), 4.53^{*}(\mathrm{~d}, \mathrm{~J}=8.8$ $\mathrm{Hz}, 1 \mathrm{H}$ ), 4.18 (brs, 1H), 3.87 (dq. J = 2.4 Hz, $6.0 \mathrm{~Hz}, 1 \mathrm{H}), 3.60$ (s, 3H), 3.58* (s, 3H), $2.92(\mathrm{dd}, \mathrm{J}=2.4 \mathrm{~Hz}, 8.8 \mathrm{~Hz}, 1 \mathrm{H}), 2.87^{*}(\mathrm{~s}, 3 \mathrm{H}), 2.66(\mathrm{~s}, 3 \mathrm{H}), 2.57-2.48(\mathrm{~m}, 1 \mathrm{H}), 2.30-$ $2.11(\mathrm{~m}, 2 \mathrm{H}), 1.77(\mathrm{dd}, \mathrm{J}=8.0,13.6 \mathrm{~Hz}, 1 \mathrm{H}), 1.43^{*}(\mathrm{~s}, 3 \mathrm{H}), 1.42(\mathrm{~s}, 3 \mathrm{H}), 1.16$ (d, J = 7.2 $\mathrm{Hz}, 3 \mathrm{H}), 1.04(\mathrm{~s}, 9 \mathrm{H}), 0.95(\mathrm{~d}, \mathrm{~J}=6.0 \mathrm{~Hz}, 3 \mathrm{H}), 0.86(\mathrm{~d}, \mathrm{~J}=6.4 \mathrm{~Hz}, 3 \mathrm{H}), 0.82^{*}(\mathrm{~d}, \mathrm{~J}=6.0$ $\mathrm{Hz}, 3 \mathrm{H}), 0.73(\mathrm{~d}, \mathrm{~J}=6.0 \mathrm{~Hz}, 3 \mathrm{H}) .{ }^{13} \mathrm{C} \mathrm{NMR}(2: 1$ rotamer ratio, sterisk denotes minor rotamer peaks, $\left.400 \mathrm{MHz}, \mathrm{CDCl}_{3}\right) \delta 178.0,177.0^{*}, 142.2,141.9^{*}, 135.6,134.1,133.5$, $130.9,129.9^{*}, 129.5^{*}, 129.2,128.2^{*}, 127.8,127.3,126.5^{*}, 125.9,89.4,75.8,74.9,71.3$, 71.2*, 61.9*, 61.0, 57.6, 44.3*, 43.3, 35.3, 35.1*, 34.5*, 34.3, 33.5*, 27.0*, 26.7, 18.9, $17.2,17.0^{*}, 16.6^{*}, 16.5^{*}, 16.3,15.4,15.3^{*}, 14.0$; IR (Neat) $v_{\max } 3369,2963,2930,2857$, 1618, 1451, 1427, 1381, 1200, 1044, 927, 909, 730, 699, 608; HRMS (Cl/ $\left.\mathrm{NH}_{3}\right) \mathrm{m} / z$ calcd for $\mathrm{C}_{39} \mathrm{H}_{55} \mathrm{NO}_{4} \mathrm{Si}[\mathrm{M}+\mathrm{Na}]^{+} 652.3798$ found $652.3804 ;[\alpha]^{20}{ }_{\mathrm{D}}=+59.1^{\circ}\left(c 1.3, \mathrm{CHCl}_{3}\right)$.

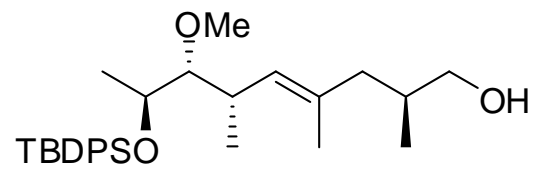

\section{$(2 S, 6 S, 7 R, 8 S, E)-8$-(tert-butyldiphenylsilyloxy)-7- methoxy-2, 4, 6-trimethylnon-4-en-1-ol (17). A} schlenk flask was charged with lithium chloride (318 $\mathrm{mg}, 7.5 \mathrm{mmol})$ and was flame dried under high vacuum. Freshly distilled diisopropylamine $(736 \mu \mathrm{L}, 5.25 \mathrm{mmol})$ and anhydrous THF $(0.23 \mathrm{M}, 5.4$ $\mathrm{mL})$ were added in. $\mathrm{n}$-BuLi $(2.50 \mathrm{M}$ in hexanes, $1.95 \mathrm{~mL}, 4.87 \mathrm{mmol})$ was charged in at $78^{\circ} \mathrm{C}$. The mixture was warmed up to RT and was stirred at that temperature for another $15 \mathrm{~min}$ before being cooled back to $0^{\circ} \mathrm{C}$. $\mathrm{BH}_{3} \cdot \mathrm{NH}_{3}$ complex $(90 \%$ tech. $171 \mathrm{mg}, 5 \mathrm{mmol})$ was added in. The reaction mixture was stirred at $0{ }^{\circ} \mathrm{C}$ for $15 \mathrm{~min}$ before being warmed up to $\mathrm{rt}$ and stirred at $\mathrm{rt}$ for another $15 \mathrm{~min}$. The mixture was cooled back to $0{ }^{\circ} \mathrm{C}$ and a solution of methyl amide 16 (787mg, $1.25 \mathrm{mmol}, 0.2 \mathrm{M}$ in THF) was added in dropwise. The reaction was allowed to stir at $\mathrm{rt}$ for $2 \mathrm{~h}$ before being quenched with $1 \mathrm{~N} \mathrm{HCl}$ at $0{ }^{\circ} \mathrm{C}$ until the $\mathrm{pH}$ of aqueous layer was 7.0. The aqueous layer was extracted with diethyl ether (3X). The combined organic layers were washed with brine, dried over anhydrous $\mathrm{MgSO}_{4}$, and were concentrated under pressure to give a yellow residue. Column chromatography on silica gel $(20 \%$ EtOAc/hexane eluant) gave viscous pale yellow oil. (496mg, 85\%, dr > 10:1). ${ }^{1} \mathrm{H}$ NMR (400 MHz, $\left.\mathrm{CDCl}_{3}\right) \delta 7.67-7.64(\mathrm{~m}, 4 \mathrm{H}), 7.42-7.33$ $(\mathrm{m}, 6 \mathrm{H}), 4.63(\mathrm{~d}, \mathrm{~J}=10 \mathrm{~Hz}, 1 \mathrm{H}), 3.89(\mathrm{dq}, \mathrm{J}=2.0 \mathrm{~Hz}, 6.0 \mathrm{~Hz}, 1 \mathrm{H}), 3.60(\mathrm{~s}, 3 \mathrm{H}), 3.40-$ $3.28(\mathrm{~m}, 2 \mathrm{H}), 2.92(\mathrm{dd}, \mathrm{J}=2.8,9.2 \mathrm{~Hz}), 2.31-2.20(\mathrm{~m}, 1 \mathrm{H}), 1.90(\mathrm{dd}, \mathrm{J}=6.8,13.2 \mathrm{~Hz}$, $1 \mathrm{H}), 1.68(\mathrm{~m}, 1 \mathrm{H}), 1.57-1.52(\mathrm{~m}, 1 \mathrm{H}), 1.43(\mathrm{~s}, 3 \mathrm{H}), 1.38(\mathrm{brs}, \mathrm{OH}), 1.04(\mathrm{~s}, 9 \mathrm{H}), 0.96(\mathrm{~d}$, $\mathrm{J}=6.4,3 \mathrm{H}), 0.86(\mathrm{~d}, \mathrm{~J}=6.8 \mathrm{~Hz}, 3 \mathrm{H}), 0.61(\mathrm{~d}, \mathrm{~J}=6.4 \mathrm{~Hz}, 3 \mathrm{H}) ;{ }^{13} \mathrm{C} \mathrm{NMR}(400 \mathrm{MHz}$, $\left.\mathrm{CDCl}_{3}\right) \delta 136.0,134.5,134.1,132.7,129.6,127.5,89.9,71.6,68.3,61.3,44.1,35.5,33.6$, 27.0, 19.1, 17.6, 16.6, 16.4, 15.8; IR (Neat) $v_{\max } 3355,3071,3049,2957,2930,2858$, 1472, 1428, 1383, 1103, 1043, 931, 822, 739, 702; HRMS $\left(\mathrm{Cl} / \mathrm{NH}_{3}\right) \mathrm{m} / \mathrm{z}$ calcd for $\mathrm{C}_{29} \mathrm{H}_{44} \mathrm{O}_{3} \mathrm{Si}[\mathrm{M}+\mathrm{Na}]^{+} 491.2957$ found 491.2981. $[\alpha]^{20}{ }_{\mathrm{D}}=+8.9^{\circ}\left(c 1.1, \mathrm{CHCl}_{3}\right)$. 
<smiles>CO[C@H](C)[C@@H](C)/C=C(\C)C[C@H](C)/C=C/I</smiles>

tert-butyl $((2 S, 3 R, 4 S, \quad 5 E, \quad 8 S, \quad 9 E)$-10-iodo-3methoxy-4,6,8-trimethyldeca-5,9-dien-2yloxy)diphenylsilane (8). A solution of oxalyl chloride $(230 \mu \mathrm{L}, 2.62 \mathrm{mmol})$ in dry DCM $(0.2 \mathrm{M}, 5 \mathrm{~mL})$ was cooled to $-78{ }^{\circ} \mathrm{C}$ and a solution of DMSO $(337 \mu \mathrm{L}, 4.72 \mathrm{mmol})$ in $4 \mathrm{~mL}$ DCM was charged in. The mixture was stirred at $-78{ }^{\circ} \mathrm{C}$ for $15 \mathrm{~min}$ before a solution of primary alcohol 17 (490mg, $1.05 \mathrm{mmol}$ ) in $3.5 \mathrm{~mL}$ DCM was added dropwisely. The reaction mixture was stirred at that temperature for 30min before being quenched with triethyl amine $(730 \mu \mathrm{L}, 5.25 \mathrm{mmol})$. The reaction mixture was warmed up to $0{ }^{\circ} \mathrm{C}$ and stirred for another 30min at that temperature before saturated ammonium chloride solution was added into the heterogeneous reaction mixture. The aqueous layer was extracted with diethyl ether $(3 \mathrm{X})$. The combined organic layers were combined, washed with brine and concentrated under pressure to give yellow oil as the crude product (quantitative crude yield). The crude aldehyde was used for the next step without further purification. Crude ${ }^{1} \mathrm{H}$ NMR $\left(400 \mathrm{MHz}, \mathrm{CDCl}_{3}\right) \delta 9.50(\mathrm{~d}, \mathrm{~J}=1.6 \mathrm{~Hz}, 1 \mathrm{H}), 7.67-7.64(\mathrm{~m}, 4 \mathrm{H}), 7.42-7.33$ $(\mathrm{m}, 6 \mathrm{H}), 4.66(\mathrm{~d}, \mathrm{~J}=9.6 \mathrm{~Hz}, 1 \mathrm{H}), 3.85(\mathrm{dd}, \mathrm{J}=2.4,6.0 \mathrm{~Hz}, 1 \mathrm{H}), 3.60(\mathrm{~s}, 3 \mathrm{H}), 2.92(\mathrm{dd}, \mathrm{J}$ $=2.4,8.8 \mathrm{~Hz}, 1 \mathrm{H}), 2.35-2.22(\mathrm{~m}, 3 \mathrm{H}), 1.72(\mathrm{dd}, \mathrm{J}=8.8,13.6 \mathrm{~Hz}, 1 \mathrm{H}), 1.44(\mathrm{~s}, 3 \mathrm{H}), 1.04$ $(\mathrm{s}, 9 \mathrm{H}), 0.97(\mathrm{~d}, \mathrm{~J}=6.0 \mathrm{~Hz}, 3 \mathrm{H}), 0.86(\mathrm{~d}, \mathrm{~J}=6.4 \mathrm{~Hz}, 3 \mathrm{H}), 0.74(\mathrm{~d}, \mathrm{~J}=7.2 \mathrm{~Hz}, 3 \mathrm{H})$.

A schlenk flask was charged with $\mathrm{CrCl}_{2}$ (flammable dried under high vacuum, $886 \mathrm{mg}, 7.2 \mathrm{mmol}$ ) in $7 \mathrm{~mL}$ anhydrous dioxane/THF (6:1 mixture). In another flask, the crude aldehyde $(1.05 \mathrm{mmol})$ and $\mathrm{CHI}_{3}(1.06 \mathrm{~g}, 2.7 \mathrm{mmol})$ was purged with argon three times before $4 \mathrm{~mL}$ dioxane/THF (6:1 mixture) was added in. The aldehyde and iodoform solution was transferred into the $\mathrm{CrCl}_{2}$ solution slowly under argon gas at $0{ }^{\circ} \mathrm{C}$. The dark green reaction mixture was covered with aluminum foil and was stirred at $\mathrm{rt}$ overnight before being diluted with diethyl ether $(5 \mathrm{~mL})$ and quenched at $0{ }^{\circ} \mathrm{C}$ with water $(10 \mathrm{~mL})$. The aqueous layer was extracted with diethyl ether $(3 \mathrm{X})$. The organic phases were combined, washed with brine and saturated thiosulfate solution and concentrated under pressure to give a yellow residue. Column chromatography on silica gel (2\% EtOAc/hexane eluant) gave the product as clear oil $\left(381 \mathrm{mg}, 75 \%\right.$ yield in 2 steps). ${ }^{1} \mathrm{H}$ NMR $\left(400 \mathrm{MHz}, \mathrm{CDCl}_{3}\right) \delta 7.70-7.64(\mathrm{~m}, 4 \mathrm{H}), 7.43-7.33(\mathrm{~m}, 6 \mathrm{H}), 6.27(\mathrm{dd}, \mathrm{J}=8.0$, $14.4 \mathrm{~Hz}, 1 \mathrm{H}), 5.76(\mathrm{~d}, \mathrm{~J}=14.4 \mathrm{~Hz}, 1 \mathrm{H}), 4.55(\mathrm{~d}, \mathrm{~J}=9.6 \mathrm{~Hz}, 1 \mathrm{H}), 3.86(\mathrm{dd}, \mathrm{J}=2.0,6.0 \mathrm{~Hz}$, $1 \mathrm{H}), 3.61(\mathrm{~s}, 3 \mathrm{H}), 2.92(\mathrm{dd}, \mathrm{J}=2.4 \mathrm{~Hz}, 8.8 \mathrm{~Hz}, 1 \mathrm{H}), 2.26-2.10(\mathrm{~m}, 2 \mathrm{H}), 1.74\left(\mathrm{ABq}, \mathrm{J}_{\mathrm{AB}}\right.$ $=13.8 \mathrm{~Hz}, \Delta v=30.5 \mathrm{~Hz}, 1 \mathrm{H}), 1.72(\mathrm{ABq}, \mathrm{J}=13.8 \mathrm{~Hz}, \Delta v=30.5 \mathrm{~Hz}, 1 \mathrm{H}), 1.38(\mathrm{~s}, 3 \mathrm{H})$, $1.04(\mathrm{~s}, 3 \mathrm{H}), 0.96(\mathrm{~d}, \mathrm{~J}=6.4 \mathrm{~Hz}, 3 \mathrm{H}), 0.87(\mathrm{~d}, \mathrm{~J}=6.4 \mathrm{~Hz}, 3 \mathrm{H}), 0.70(\mathrm{~d}, \mathrm{~J}=7.2 \mathrm{~Hz}, 3 \mathrm{H})$; ${ }^{13} \mathrm{C}$ NMR $\left(400 \mathrm{MHz}, \mathrm{CDCl}_{3}\right) \delta 151.7,135.9,134.4,131.6,129.6,127.4,89.8,73.1,71.7$, 61.3, 46.5, 38.9, 35.5, 27.0, 19.1, 18.8, 17.8, 16.5, 15.8; IR (Neat) $v_{\max } 3071,3049,2958$, 2929, 2857, 1589, 1472, 1427, 1381, 1098, 1042, 931, 821, 738, 698, 608; HRMS $\left(\mathrm{Cl} / \mathrm{NH}_{3}\right) \mathrm{m} / \mathrm{z}$ calcd for $\mathrm{C}_{30} \mathrm{H}_{43} \mathrm{IO}_{2} \mathrm{Si}[\mathrm{M}+\mathrm{Na}]^{+} 613.1975$ found $613.1993 ;[\alpha]^{20} \mathrm{D}=+12.5^{\circ}$ (c $\left.1.5, \mathrm{CHCl}_{3}\right)$. 
Preparation of silyl-substituted methacrolein $\mathbf{1 0}:^{2}$

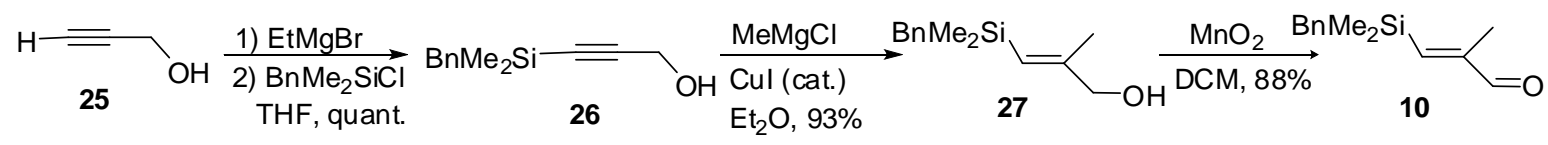

(E)-3-(benzyldimethylsilyl)-2-methylprop-2-en-1-ol (27).

${ }^{1} \mathrm{H}$ NMR $\left(400 \mathrm{MHz}, \mathrm{CDCl}_{3}\right) \delta 7.21-7.17(\mathrm{~m}, 2 \mathrm{H}), 7.07-7.03(\mathrm{~m}$, $1 \mathrm{H}), 7.00-6.98(\mathrm{~m}, 2 \mathrm{H}), 5.47(\mathrm{~s}, 1 \mathrm{H}), 3.99(\mathrm{~d}, \mathrm{~J}=6.4 \mathrm{~Hz}, 2 \mathrm{H})$, $2.17(\mathrm{~s}, 2 \mathrm{H}), 1.67(\mathrm{~s}, 3 \mathrm{H}), 0.10(\mathrm{~s}, 6 \mathrm{H}) .{ }^{13} \mathrm{C} \mathrm{NMR}\left(400 \mathrm{MHz}, \mathrm{CDCl}_{3}\right) \delta 154.23,140.0$, 128.1, 128.0, 124.0, 68.6, 26.5, 18.4, -2.0; IR (Neat) $v_{\max } 3321,2955,2896,1629,1492$, $1451,1248,1206,1152,1056,881,815$.

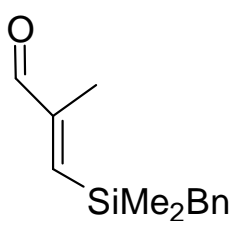

(E)-3-(benzyldimethylsilyl)-2-methylacrylaldehyde (10). A solution of allylic alcohol $27(10 \mathrm{~g}, 45 \mathrm{mmol})$ in DCM $(0.3 \mathrm{M}, 150 \mathrm{~mL})$ was added pre-activated $\mathrm{MnO}_{2} \quad(58.7 \mathrm{~g}, 675 \mathrm{mmol})$. The black heterogeneous reaction mixture was stirred at $\mathrm{rt}$ for $10 \mathrm{~min}$ before being filtered through celite to give a yellow oil as crude product. The crude aldehyde was used without any further purification $(8.87 \mathrm{~g}, 89 \%$ crude yield). ${ }^{1} \mathrm{H}$ NMR $\left(400 \mathrm{MHz}, \mathrm{CDCl}_{3}\right) \delta 9.18(\mathrm{~s}, 1 \mathrm{H}), 7.04-6.98(\mathrm{~m}, 2 \mathrm{H}), 6.90-6.86$ $(\mathrm{m}, 1 \mathrm{H}), 6.79-6.78(\mathrm{~m}, 2 \mathrm{H}), 6.43(\mathrm{~s}, 1 \mathrm{H}), 2.05(\mathrm{~s}, 2 \mathrm{H}), 1.58(\mathrm{~s}, 3 \mathrm{H}), 0.00(\mathrm{~s}, 6 \mathrm{H}) ;{ }^{13} \mathrm{C}$ NMR $\left(400 \mathrm{MHz}, \mathrm{CDCl}_{3}\right) \delta 195.7,153.0,151.1,138.7,128.3,124.4,25.5,13.5,-2.7$; IR (Neat) $v_{\max } 3060,2694,2897,3024,2799,2956,1692,1599,1493,1452,1333,1250$, 1207, 1151,1057, 1019, 905, 841.7, 794,763, 699, 629.

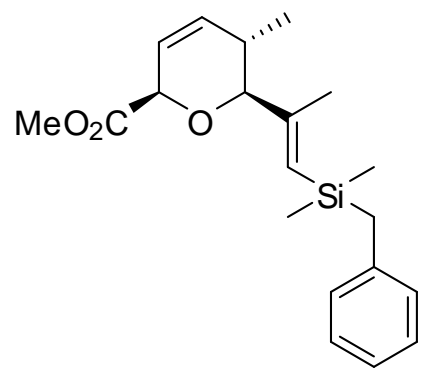

$(2 R, 5 S, 6 S)$-methyl 6-((E)-1-(benzyldimethylsilyl)prop-1en-2-yl)-5-methyl-5,6-dihydro-2H-pyran-2-carboxylate (18). A solution of crotylsilane $9(1.94 \mathrm{~g}, 5.5 \mathrm{mmol})$ and aldehyde 10 (1.33g, $6.1 \mathrm{mmol})$ in MeCN/DCM (3:1 mixture) $(0.05 \mathrm{M}, 108 \mathrm{~mL})$ was cooled to $-78^{\circ} \mathrm{C}$. Di-tert-butyl pyridine $(185 \mu \mathrm{L}, 0.82 \mathrm{mmol})$ was charged in at that temperature followed by slow addition of TMSOTf $(1.06 \mathrm{~mL}, 5.5 \mathrm{mmol})$.

The yellow reaction mixture was warmed up to $-20{ }^{\circ} \mathrm{C}$ and stirred for another $3 \mathrm{~h}$ before being quenched with saturated $\mathrm{NaHCO}_{3}$ solution $(50 \mathrm{~mL})$ at $20{ }^{\circ} \mathrm{C}$ and slowly warmed up to RT. The aqueous layer was extracted with diethyl ether $(3 \mathrm{X})$. The combined organic layers were washed with brine, dried over anhydrous $\mathrm{MgSO}_{4}$ and concentrated under pressure to give a brown residue. Column chromatography on silica gel (5\% EtOAc/hexane eluant) gave the product as pale yellow oil $(1.12 \mathrm{~g}, 65 \%)$. (2\% EtOAc/hexanes eluent gave unreacted aldehyde 395mg and $20 \%$ EtOAc/hexanes gave deprotected crotylsilane 335mg). ${ }^{1} \mathrm{H}$ NMR (400 $\left.\mathrm{MHz}, \mathrm{CDCl}_{3}\right) \delta$ 7.19 - $6.98(\mathrm{~m}, 5 \mathrm{H}), 5.77(\mathrm{~s}, 2 \mathrm{H}), 5.39(\mathrm{~s}, 1 \mathrm{H}), 4.79(\mathrm{~d}, \mathrm{~J}=3.6 \mathrm{~Hz}, 1 \mathrm{H}), 3.74(\mathrm{~s}, 3 \mathrm{H}), 3.52$ $(\mathrm{d}, \mathrm{J}=9.2 \mathrm{~Hz}, 1 \mathrm{H}), 2.34-2.16(\mathrm{~m}, 1 \mathrm{H}), 2.16(\mathrm{~s}, 2 \mathrm{H}), 1.79(\mathrm{~s}, 3 \mathrm{H}), 0.81(\mathrm{~d}, \mathrm{~J}=6.8 \mathrm{~Hz}$, $3 \mathrm{H}), 0.105(\mathrm{~d}, \mathrm{~J}=7.2 \mathrm{~Hz}, 3 \mathrm{H}), 0.097(\mathrm{~d}, \mathrm{~J}=7.2 \mathrm{~Hz}, 3 \mathrm{H}) ;{ }^{13} \mathrm{C} \mathrm{NMR}\left(400 \mathrm{MHz}, \mathrm{CDCl}_{3}\right) \delta$ $170.5,152.2$, 140.0, 133.7, 128.2, 126.5, 124.0, 123.1, 88.4, 74.5, 52.3, 31.0, 26.4, 17.0, -

\footnotetext{
${ }^{2}$ Detailed experimental procedures for prepration of 26 and 27, see: (a) Mohamed, M.; Brook, M. A. Helv. Chim. Acta. 2002, 85, 4165. (b) Spino, C.; Gobdout C. J. Am. Chem. Soc. 2003, 125, 12106.
} 
2.0; IR (Neat) $v_{\max } 3059,3024,2953,2927,2852,1619,1600,1493,1452,1378,1248$, 1207, 1155, 1102, 1071, 1017, 833, 699; HRMS $\left(\mathrm{Cl} / \mathrm{NH}_{3}\right) \mathrm{m} / z$ calcd for $\mathrm{C}_{20} \mathrm{H}_{28} \mathrm{O}_{3} \mathrm{Si}$ $[\mathrm{M}+\mathrm{Na}]^{+} 367.1705$, found $367.1710 ;[\alpha]^{20}{ }_{\mathrm{D}}=-111.2^{\circ}\left(c 1.1, \mathrm{CHCl}_{3}\right)$.

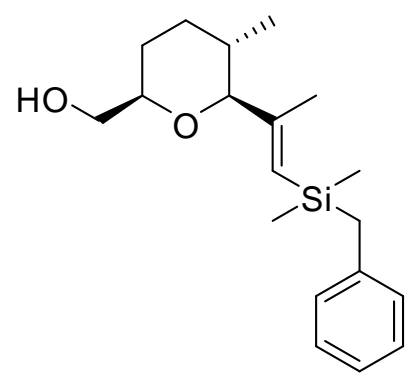

((5S)-6-((E)-1-(benzyldimethylsilyl)prop-1-en-2-yl)-5methyltetrahydro-2H-pyran-2-yl)methanol (19). A solution of methyl ester 18 (454mg, $1.32 \mathrm{mmol})$ in diethyl ether $(44 \mathrm{~mL}$, $0.03 \mathrm{M}$ ) was cooled to $0{ }^{\circ} \mathrm{C}$, LAH (106mg, $\left.2.64 \mathrm{mmol}\right)$ was charged in. The heterogeneous reaction was stirred at that temperature for $40 \mathrm{~min}$ before being quenched with $5 \% \mathrm{HCl}$ solution carefully. The aqueous layer was extracted with diethyl ether $(3 \mathrm{X})$. The combined organic layers were washed with brine, dried over anhydrous $\mathrm{MgSO}_{4}$, and concentrated under pressure to give viscous clear oil as the crude product. The compound was subjected to the next step without further purification.

A solution of homoallylic alcohol (393mg, $1.24 \mathrm{mmol})$ in ethanol $(12 \mathrm{~mL}, 0.1 \mathrm{M})$ was stirred vigorously at RT, chlorotris(triphenylphosphine)rhodium (11 $\mathrm{mg}, 0.12 \mathrm{mmol}$ ) was added. The yellow reaction mixture was stirred at $\mathrm{rt}$ under $\mathrm{H}_{2}(75 \mathrm{psi})$ before it was checked by crude NMR of the reaction completeness ( $c a$. 16h). The orange reaction mixture was concentrated under pressure to give a dark brown residue. Column chromatography on silica gel (15\% EtOAc/hexane eluant) gave the product as viscous pale yellow oil $(350 \mathrm{mg}$, $84 \%$ in 2 steps). ${ }^{1} \mathrm{H}$ NMR $\left(400 \mathrm{MHz}, \mathrm{CDCl}_{3}\right) \delta 7.19-6.98(\mathrm{~m}, 5 \mathrm{H}), 5.32(\mathrm{~s}, 1 \mathrm{H}), 3.53$ $\left(\mathrm{ABq}, \mathrm{J}_{\mathrm{AB}}=2.0 \mathrm{~Hz}, \Delta v=27.4 \mathrm{~Hz}, 2 \mathrm{H}\right), 3.31(\mathrm{~d}, \mathrm{~J} 9.2 \mathrm{~Hz}, 1 \mathrm{H}), 3.50-3.34(\mathrm{~m}, 1 \mathrm{H}), 2.16$ (s, 2H), 1.89-1.81 (m, 1H), $1.68(\mathrm{~s}, 3 \mathrm{H}), 1.51-1.14(\mathrm{~m}, 4 \mathrm{H}), 0.68(\mathrm{~d}, \mathrm{~J}=6 \mathrm{~Hz}, 3 \mathrm{H})$, 0.090 (s, 3H), 0.086 (s, 3H); ${ }^{13} \mathrm{C}$ NMR $\left(400 \mathrm{MHz}, \mathrm{CDCl}_{3}\right) \delta 153.5,139.9,128.0,127.9$, 125.4, 123.8, 92.2, 77.6, 66.0, 32.4, 31.9, 27.5, 26.3, 17.6, 16.8, -2.0; IR (Neat) $v_{\max } 3430$, 3059, 3024, 2951, 2924, 2850, 1618, 1600, 1493, 1452, 1377, 1247, 1207, 1153, 1107, 1073, 1045, 836, 763, 698; HRMS $\left(\mathrm{Cl} / \mathrm{NH}_{3}\right) \mathrm{m} / \mathrm{z}$ calcd for $\mathrm{C}_{19} \mathrm{H}_{30} \mathrm{O}_{2} \mathrm{Si}[\mathrm{M}+\mathrm{Na}]^{+} 341.1913$, found $341.1902 ;[\alpha]^{20}{ }_{\mathrm{D}}=-3.5^{\circ}\left(c 1.3, \mathrm{CHCl}_{3}\right)$.

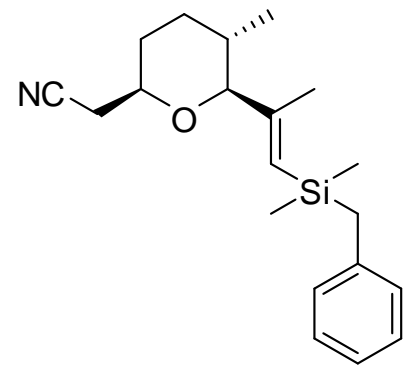

\section{2-((5S)-6-((E)-1-(benzyldimethylsilyl)prop-1-en-2-yl)-5- methyltetrahydro-2H-pyran-2-yl)acetonitrile (20). A} solution of alcohol 19 (268mg, $0.84 \mathrm{mmol})$ in DCM $(2.8 \mathrm{~mL}$, $0.3 \mathrm{M})$ was stirred at rt. After which pyridine $(340 \mu \mathrm{L}, 4.21$ mmol), $p$-toluenesulfonyl chloride $(642 \mathrm{mg}, 3.36 \mathrm{mmol})$ and DMAP (21mg, $0.17 \mathrm{mmol})$ were added into the reaction. The homogeneous reaction mixture was stirred at that temperature over night before being quenched with $\mathrm{H}_{2} \mathrm{O}(2 \mathrm{~mL})$. The inorganic layer was extracted with diethyl ether $(3 \mathrm{X})$. The combined organic layers were washed with brine, dried over anhydrous magnesium sulfate and concentrated under pressure to give the crude product as yellow oil.

To the crude tosylate product, DMF $(0.05 \mathrm{M}, 17 \mathrm{~mL}$ ) and sodium cynide (206 mg, 4.2 mmol) were added. The yellow reaction mixture was stirred under reflux for $2 \mathrm{~h}$ before being quenched with water $5 \mathrm{~mL}$ at $\mathrm{rt}$. The inorganic layer was extracted with diethyl ether (3X). The combined organic layers were washed with brine, dried over anhydrous magnesium sulfate and concentrated under pressure to give a yellow residue. Column chromatography on silica gel (10\% EtOAc in hexane eluent) gave the product as a pale 
yellow oil (66\% in 2 steps). ${ }^{1} \mathrm{H}$ NMR $\left(400 \mathrm{MHz}, \mathrm{CDCl}_{3}\right) \delta 7.19-6.98(\mathrm{~m}, 5 \mathrm{H}), 5.32(\mathrm{~s}$, $1 \mathrm{H}), 3.61-3.57(\mathrm{~m}, 1 \mathrm{H}), 3.32(\mathrm{~d}, \mathrm{~J}=10.0 \mathrm{~Hz}, 1 \mathrm{H}), 2.53(\mathrm{~d}, \mathrm{~J}=5.6 \mathrm{~Hz}, 2 \mathrm{H}), 2.16(\mathrm{~s}, 2 \mathrm{H})$, $1.90-1.85(\mathrm{~m}, 1 \mathrm{H}), 1.76-1.72(\mathrm{~m}, 1 \mathrm{H}), 1.69(\mathrm{~s}, 3 \mathrm{H}), 1.47-1.44(\mathrm{~m}, 2 \mathrm{H}), 1.26-1.18(\mathrm{~m}$, $2 \mathrm{H}), 0.68(\mathrm{~d}, \mathrm{~J}=6.4 \mathrm{~Hz}, 3 \mathrm{H}), 0.09(\mathrm{~s}, 3 \mathrm{H}), 0.08(\mathrm{~s}, 3 \mathrm{H}) ;{ }^{13} \mathrm{C} \mathrm{NMR}\left(400 \mathrm{MHz}, \mathrm{CDCl}_{3}\right) \delta$ 153.1, 140.0, 128.2, 128.1, 125.9, 124.0, 117.3, 92.7, 72.1, 31.8, 31.0, 29.6, 26.4, 24.8, 17.5, 16.6, -2.0; IR (Neat) $v_{\max } 3080,3059,2953,2927,2852,1619,1600,1493,1452$, 1378, 1248, 1207, 1155, 1102, 1071, 1017, 833, 699; HRMS $\left(\mathrm{Cl} / \mathrm{NH}_{3}\right) \mathrm{m} / z$ calcd for $\mathrm{C}_{20} \mathrm{H}_{29} \mathrm{NOSi}[\mathrm{M}+\mathrm{Na}]^{+} 350.1916$, found 350.1927. $[\alpha]^{20}{ }_{\mathrm{D}}=-18.8\left(c\right.$ 1.5, $\left.\mathrm{CHCl}_{3}\right)$.

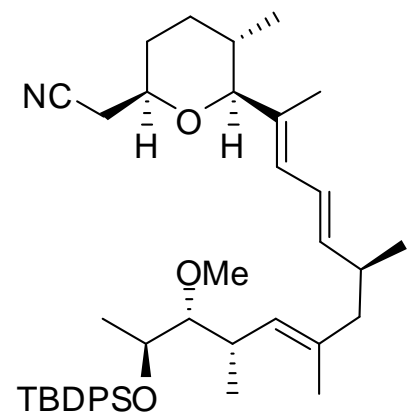

2-((2R,5S,6S)-6-((2E,4E,6S,8E,10S,11R,12S)-12-(tertbutyldiphenylsilyloxy)-11-methoxy-6,8,10-trimethyltrideca2,4,8-trien-2-yl)-5-methyltetrahydro-2H-pyran-2yl)acetonitrile (21). A solution of vinylsilane $20(71 \mathrm{mg}, 0.22$ $\mathrm{mmol})$ in $\mathrm{THF}(0.1 \mathrm{M}, 2.2 \mathrm{~mL})$ was stirred at $0{ }^{\circ} \mathrm{C}$. TBAF $(1.0$ $\mathbf{M}$ in THF, $0.48 \mathrm{~mL}$ ) was slowly added into the reaction. The orange reaction mixture was stirred at $0{ }^{\circ} \mathrm{C}$ for $5 \mathrm{~min}$ and at $\mathrm{rt}$ for $10 \mathrm{~min}$. The reaction was cooled back to $0{ }^{\circ} \mathrm{C}$ and $[\text { AllyPdCl }]_{2}(4 \mathrm{mg}, 1 \mu \mathrm{mol})$ was added into the reaction mixture followed by slow addition of vinyl iodide 8 (135mg, $0.24 \mathrm{mmol}$, in $3 \mathrm{~mL}$ THF). The reaction was covered with foil and was stirred at $\mathrm{rt}$ for $6 \mathrm{~h}$. The dark brown reaction was passed through a short column of silicon gel before the solvent was evaporated off to give a dark brown residue. Column chromatography on silicon gel with $2 \%$ EtOAc/hexanes recovered part of the unreacted vinyl iodide $(50 \mathrm{mg}), 8 \% \mathrm{EtOAc} /$ hexanes removed minor impurities and 10\% EtOAc/hexanes gave the product as yellow oil. (72 $\mathrm{mg}, 51 \%$ iso $64 \%$ based on $85 \%$ conversion, in cases which were under $30 \mathrm{mg}$ scale of each starting material, isolated yields were around $70 \%$ with over $90 \%$ conversion of vinyl iodide $)^{1} \mathrm{H}$ NMR (400 MHz, $\left.\mathrm{CDCl}_{3}\right) \delta 7.67-7.64(\mathrm{~m}, 4 \mathrm{H}), 7.43-7.32(\mathrm{~m}, 6 \mathrm{H}), 6.10(\mathrm{dd}, \mathrm{J}=11.2$, $14.4 \mathrm{~Hz}, 1 \mathrm{H}), 5.89(\mathrm{~d}, \mathrm{~J}=11.6 \mathrm{~Hz}, 1 \mathrm{H}), 5.49(\mathrm{dd}, \mathrm{J}=7.2,15.6 \mathrm{~Hz}, 1 \mathrm{H}), 4.59$ (d, J = 9.6 $\mathrm{Hz}, 1 \mathrm{H}), 3.91(\mathrm{dq}, \mathrm{J}=2.4,6.4 \mathrm{~Hz}, 1 \mathrm{H}), 3.60(\mathrm{~s}, 3 \mathrm{H}), 3.65-3.56(\mathrm{~m}, 1 \mathrm{H}), 3.32(\mathrm{~d}, \mathrm{~J}=9.6$ $\mathrm{Hz}, 1 \mathrm{H}), 2.91(\mathrm{dd}, \mathrm{J}=2.4,8.8 \mathrm{~Hz}, 1 \mathrm{H}), 2.53(\mathrm{~d}, \mathrm{~J}=5.6 \mathrm{~Hz}, 2 \mathrm{H}), 2.28-2.15(\mathrm{~m}, 2 \mathrm{H})$, $1.92-1.87(\mathrm{~m}, 2 \mathrm{H}), 1.79-1.73(\mathrm{~m}, 1 \mathrm{H}), 1.70(\mathrm{~s}, 3 \mathrm{H}), 1.65-1.60(\mathrm{~m}, 1 \mathrm{H}), 1.50-1.46$ $(\mathrm{m}, 1 \mathrm{H}), 1.40(\mathrm{~s}, 3 \mathrm{H}), 1.27-1.19(\mathrm{~m}, 2 \mathrm{H}), 1.00(\mathrm{~s}, 9 \mathrm{H}), 0.95(\mathrm{~d}, \mathrm{~J}=6.4 \mathrm{~Hz}, 3 \mathrm{H}), 0.85(\mathrm{~d}$, $\mathrm{J}=6.4 \mathrm{~Hz}, 3 \mathrm{H}), 0.70(\mathrm{~d}, \mathrm{~J}=6.8 \mathrm{~Hz}, 3 \mathrm{H}), 0.64(\mathrm{~d}, \mathrm{~J}=6.8 \mathrm{~Hz}, 3 \mathrm{H}) ;{ }^{13} \mathrm{C} \mathrm{NMR}(400 \mathrm{MHz}$, $\left.\mathrm{CDCl}_{3}\right) \delta 141.0,136.0,133.6,129.6,128.8,127.5,127.4,123.5,117.3,90.7,89.9,72.3$, 71.7, 61.3, 47.4, 35.5, 34.7, 32.0, 31.0, 29.7, 27.0, 24.8, 22.7, 19.4, 17.5, 16.6, 15.8, 14.1, 12.1; IR (Neat) $v_{\max } 2956,2855,1455,1427,1380,1102,1069,966,932,822,702,610$; HRMS $\left(\mathrm{Cl} / \mathrm{NH}_{3}\right) \mathrm{m} / \mathrm{z}$ calcd for $\mathrm{C}_{41} \mathrm{H}_{59} \mathrm{NO}_{3} \mathrm{Si}[\mathrm{M}+\mathrm{Na}]^{+}$664.4162, found 664.4116. $[\alpha]^{20}{ }_{\mathrm{D}}$ $=-31.5^{\circ}\left(c 1.2, \mathrm{CHCl}_{3}\right) ;[\alpha]^{20}{ }_{\mathrm{D}}=-22.4^{\circ}\left(c 0.5, \mathrm{CHCl}_{3}\right)$. 


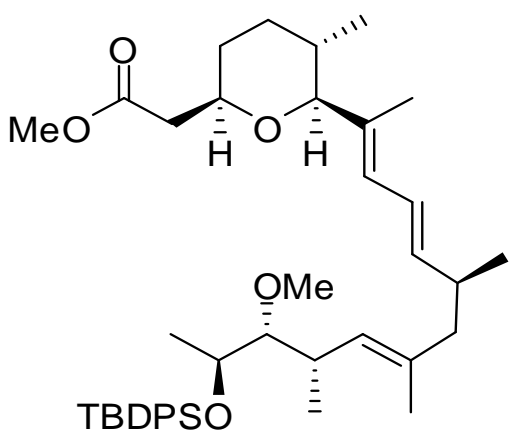

methyl

2-((2R,5S,6S)-6-

$((2 E, 4 E, 6 S, 8 E, 10 S, 11 R, 12 S)-12$-(tertbutyldiphenylsilyloxy)-11-methoxy-6,8,10trimethyltrideca-2,4,8-trien-2-yl)-5methyltetrahydro-2H-pyran-2-yl)acetate (22). A solution of nitrile $(38 \mathrm{mg}, 0.06 \mathrm{mmol})$ in dry DCM (0.05 M, $1.2 \mathrm{~mL}$ ) was cooled to $-78{ }^{\circ} \mathrm{C}$. Slowly treated the reaction with fresh DIBAL-H (1.0 $\mathbf{M}$ in hexanes, $78 \mu \mathrm{L}, 0.078 \mathrm{mmol})$. The reaction mixture was gradually warmed up to $0^{\circ} \mathrm{C}(\mathrm{ca}$. 2h) and stirred at $0{ }^{\circ} \mathrm{C}$ for another $1 \mathrm{~h}$. The reaction was quenched by addition of $\mathrm{NaF}(13 \mathrm{mg}, 0.3 \mathrm{mmol})$ and saturated ammonium chloride soln. $(2 \mathrm{~mL})$ at $0{ }^{\circ} \mathrm{C}$ after checking the reaction completeness by TLC. Further stirring was continued till the reaction mixture was well separated into two layers. The inorganic layer was extracted with diethyl ether. The combined organic layers were washed with brine, dried over anhydrous magnesium sulfate and concentrated under pressure to give yellow oil as crude product. The crude aldehyde was immediately used for the next oxidation step without any further purification.

The crude aldehyde $(28 \mathrm{mg}, 0.043 \mathrm{mmol})$ was dissolved in $t$-butyl alcohol $(0.05 \mathrm{M}, 1$ $\mathrm{mL}$ ). The mixture was cooled to $0{ }^{\circ} \mathrm{C}$ and 2-methyl butene was added quickly into the reaction followed by slow addition of freshly prepared $\mathrm{NaClO}_{2}-\mathrm{NaH}_{2} \mathrm{PO} 4-\mathrm{H}_{2} \mathrm{O}(1 \mathrm{~g}-1 \mathrm{~g}-10$ $\mathrm{mL})$ solution $(1 \mathrm{~mL})$. The yellow reaction mixture was stirred at $\mathrm{rt}$ for $3 \mathrm{~h}$ before being concentrated under pressure. The residue was diluted with diethyl ether water $(c a .1 \mathrm{~mL}$ each). The inorganic layer was acidified with $1 \mathrm{~N} \mathrm{HCl}$ till $\mathrm{PH}=4.0$. The inorganic layer was extracted with diethyl ether (3X). The combined organic layers were dried over anhydrous Magsesium sulfate and concentrated under pressure to give cloudy oil as the crude product. The crude acid was used for the next methylation step without any further purification.

The crude acid (28mg, $0.043 \mathrm{mmol})$ was dissolved in dry benzene $(0.02 \mathbf{M}, 3 \mathrm{~mL})$. Anhydrous methanol $(200 \mu \mathrm{L})$ was added followed by slowly addition of $\mathrm{TMSCHN}_{2}(2.0$ $\mathbf{M}$ in hexanes, $32 \mu \mathrm{L}, 65 \mu \mathrm{mol})$. The reaction mixture was stirred at $\mathrm{rt}$ for $15 \mathrm{~min}$ before being quenched with water $(2 \mathrm{~mL})$. The inorganic layer was extracted with diethyl ether (3X). The combined organic layers were washed with brine and concentrated under pressure to give pale yellow oil as the crude product. Column chromatography on silica gel $(10 \%$ EtOAc/hexanes eluent) gave the product as pale yellow oil $(17 \mathrm{mg}, 59 \%$ in 3 steps). ${ }^{1} \mathrm{H}$ NMR (400 MHz, CDCl3) $\delta 7.66-7.64(\mathrm{~m}, 4 \mathrm{H}), 7.42-7.33$ (m, 6H), 6.10 (dd, $\mathrm{J}=10.8,14.4 \mathrm{~Hz}, 1 \mathrm{H}), 5.87(\mathrm{~d}, \mathrm{~J}=10.8 \mathrm{~Hz}, 1 \mathrm{H}), 5.46(\mathrm{dd}, \mathrm{J}=7.20,15.2 \mathrm{~Hz}, 1 \mathrm{H}), 5.58$ $(\mathrm{d}, \mathrm{J}=10.0 \mathrm{~Hz}, 1 \mathrm{H}), 3.91(\mathrm{dq}, \mathrm{J}=2.0,6.0 \mathrm{~Hz}, 1 \mathrm{H}), 3.77-3.72(\mathrm{~m}, 1 \mathrm{H}), 3.64(\mathrm{~s}, 3 \mathrm{H}), 3.60$ $(\mathrm{s}, 3 \mathrm{H}), 3.31(\mathrm{~d}, \mathrm{~J}=10.0 \mathrm{~Hz}, 1 \mathrm{H}), 2.91(\mathrm{dd}, \mathrm{J}=2.4,8.8 \mathrm{~Hz}, 1 \mathrm{H}), 2.58(\mathrm{dd}, \mathrm{J}=6.0,15.2 \mathrm{~Hz}$, $1 \mathrm{H}), 2.38(\mathrm{dd}, \mathrm{J}=2.4,8.8 \mathrm{~Hz}, 1 \mathrm{H}), 2.27-2.15(\mathrm{~m}, 2 \mathrm{H}), 1.91-1.80(\mathrm{~m}, 2 \mathrm{H}), 1.68(\mathrm{~s}, 3 \mathrm{H})$, $1.61-1.59(\mathrm{~m}, 2 \mathrm{H}), 1.40(\mathrm{~s}, 3 \mathrm{H}), 1.38-1.16(\mathrm{~m}, 3 \mathrm{H}), 1.04(\mathrm{~s}, 9 \mathrm{H}), 0.95(\mathrm{~d}, \mathrm{~J}=6.4 \mathrm{~Hz}$, $3 \mathrm{H}), 0.85(\mathrm{~d}, \mathrm{~J}=6.4 \mathrm{~Hz}, 3 \mathrm{H}), 0.68(\mathrm{~d}, \mathrm{~J}=6.4 \mathrm{~Hz}, 3 \mathrm{H}), 0.64(\mathrm{~d}, \mathrm{~J}=6.8 \mathrm{~Hz}, 3 \mathrm{H}) ;{ }^{13} \mathrm{C} \mathrm{NMR}$ $\left(400 \mathrm{MHz}, \mathrm{CDCl}_{3}\right) \delta 171.9,140.5,136.0,134.3,132.4,129.6,129.5,128.3,127.5,123.7$, $90.4,89.9,73.8,71.7,61.4,51.5,47.4,41.3,35.5,34.7,32.2,31.6,30.3,27.0,19.4,19.2$, 17.7, 17.7, 16.6, 15.8, 12.3; IR (Neat) $v_{\max } 3071,2955,2928,2857,1741,1455,1382$, 
1289, 1197, 1103, 1067, 966, 932, 822; HRMS $\left(\mathrm{Cl} / \mathrm{NH}_{3}\right) \mathrm{m} / \mathrm{z}$ calcd for $\mathrm{C}_{42} \mathrm{H}_{62} \mathrm{O}_{5} \mathrm{Si}$ $[\mathrm{M}+\mathrm{Na}]^{+} 697.4264$, found 697.4229. $[\alpha]^{20}{ }_{\mathrm{D}}=-18.8^{\circ}\left(c 1.6, \mathrm{CHCl}_{3}\right)$.

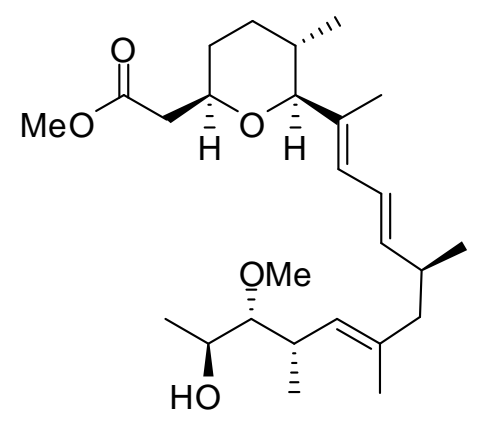

methyl 2-((2R,5S,6S)-6-((2E,4E,6S,8E,10S,11R,12S)12-hydroxy-11-methoxy-6,8,10-trimethyltrideca2,4,8-trien-2-yl)-5-methyltetrahydro-2H-pyran-2yl)acetate (23) A solution of silyl ether $22(17 \mathrm{mg}, 0.025$ $\mathrm{mmol})$ in dry THF $(0.05 \mathrm{M}, 0.5 \mathrm{~mL})$ was treated with TBAF (1.0 M in THF, $0.1 \mathrm{~mL}, 0.1 \mathrm{mmol}$ ) at rt. Further stirring was continued at that Temp for 3 days. The reaction was diluted with diethyl ether $2 \mathrm{~mL}$ followed by addition of saturated $\mathrm{NH}_{4} \mathrm{Cl}$ solution $(2 \mathrm{~mL})$. The inorganic layer was extracted with EtOAc (3X) and the combined organic layers were washed with brine, concentrated under pressure to give a yellow residue. Column chromatography on silica gel $(10 \%$ EtOAc/hexanes eluent recovered the unconverted starting material; 30\% EtOAc/hexanes) gave viscous pale yellow oil as the product $(12 \mathrm{mg}, 81 \%) .{ }^{1} \mathrm{H}$ NMR $(400 \mathrm{MHz}, \mathrm{CDCl} 3) \delta 6.16(\mathrm{dd}, \mathrm{J}=10.8$, $14.8 \mathrm{~Hz}, 1 \mathrm{H}), 5.88(\mathrm{~d}, \mathrm{~J}=10.8 \mathrm{~Hz}, 1 \mathrm{H}), 5.50(\mathrm{dd}, \mathrm{J}=6.8,15.2 \mathrm{~Hz}, 1 \mathrm{H}), 4.94(\mathrm{~d}, \mathrm{~J}=10.0$ $\mathrm{Hz}, 1 \mathrm{H}), 3.82-3.72(\mathrm{~m}, 2 \mathrm{H}), 3.64(\mathrm{~s}, 3 \mathrm{H}), 3.51(\mathrm{~s}, 3 \mathrm{H}), 3.30$ (d, J = 9.6 Hz, 1H), 2.92(dd, $\mathrm{J}=3.2,8.4 \mathrm{~Hz}, 1 \mathrm{H}), 2.58(\mathrm{dd}, \mathrm{J}=6.0,9.6 \mathrm{~Hz}, 1 \mathrm{H}), 2.46-2.33(\mathrm{~m}, 2 \mathrm{H}), 2.37(\mathrm{dd}, \mathrm{J}=6.8$, $14.8 \mathrm{~Hz}, 1 \mathrm{H}), 1.99$ (dd, J = 6.8, $13.6 \mathrm{~Hz}, 1 \mathrm{H}), 1.88$ (dd, J = 8.0, $13.6 \mathrm{~Hz}, 1 \mathrm{H}), 1.85-1.80$ $(\mathrm{m}, 1 \mathrm{H}), 1.69(\mathrm{~s}, 3 \mathrm{H}), 1.64$ (brs, OH), $1.55(\mathrm{~s}, 3 \mathrm{H}), 1.55-1.48(\mathrm{~m}, 1 \mathrm{H}), 1.36-1.15(\mathrm{~m}$, $3 \mathrm{H}), 1.08(\mathrm{~d}, \mathrm{~J}=6.4 \mathrm{~Hz}, 3 \mathrm{H}), 0.98(\mathrm{~d}, \mathrm{~J}=6.8 \mathrm{~Hz}, 3 \mathrm{H}), 0.91(\mathrm{~d}, \mathrm{~J}=6.4 \mathrm{~Hz}, 3 \mathrm{H}), 0.68(\mathrm{~d}, \mathrm{~J}$ $=6.8 \mathrm{~Hz}, 3 \mathrm{H}) ;{ }^{13} \mathrm{C} \mathrm{NMR}\left(400 \mathrm{MHz}, \mathrm{CDCl}_{3}\right) \delta 171.8,140.3,134.3,132.9,129.0,128.3$, 124.1, 90.4, 89.5, 73.8, 69.4, 61.5, 51.5, 47.3, 41.2, 35.7, 34.9, 32.3, 32.2, 31.6, 19.9, 17.8, 17.7, 17.1, 16.3, 12.3; IR (Neat) $v_{\max } 3470,2954,2926,2870,1741,1454,1379,1291$, 1160, 1198, 1098, 1067, 1019, 966; HRMS $\left(\mathrm{Cl} / \mathrm{NH}_{3}\right) \mathrm{m} / \mathrm{z}$ calcd for $\mathrm{C}_{26} \mathrm{H}_{44} \mathrm{O}_{5}[\mathrm{M}+\mathrm{Na}]^{+}$ 459.3086, found 459.3077. $[\alpha]^{20}{ }_{\mathrm{D}}=-53.2^{\circ}\left(c 0.5, \mathrm{CHCl}_{3}\right)$.

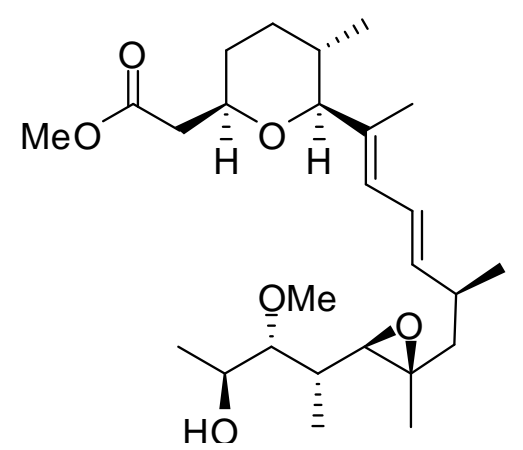

methyl 2-((2R,5S,6S)-6-((S,2E,4E)-7-((2R,3R)-3$((2 R, 3 R, 4 S)-4-h y d r o x y-3-m e t h o x y p e n t a n-2-y l)-2-$ methyloxiran-2-yl)-6-methylhepta-2,4-dien-2-yl)-5methyltetrahydro-2H-pyran-2-yl)acetate (24) A solution of bis-homoallylic alcohol $23(10 \mathrm{mg}, 0.023$ $\mathrm{mmol})$ in dry DCM $(0.025 \mathrm{M}, 1 \mathrm{~mL})$ and $\mathrm{MS}(4 \AA \hat{)})$ were stirred at $0{ }^{\circ} \mathrm{C}$. The first portion of $\mathrm{VO}(\mathrm{acac})_{2}$ (0.6mg, $2.26 \mu \mathrm{mol})$ was added followed by the addition of $t$ - $\mathrm{BuOOH}(5.5 \mathrm{M}$ in decane, $18 \mu \mathrm{L})$ dropwisely. The dark brown reaction was stirred at $0{ }^{\circ} \mathrm{C}$ for $12 \mathrm{~h}$ before a second portion of $\mathrm{VO}(\mathrm{acac})_{2}(0.6 \mathrm{mg}, 2.26 \mu \mathrm{mol})$ was added followed by slow addition of $t$ - $\mathrm{BuOOH}(5.5 \mathrm{M}$ in decane, $18 \mu \mathrm{L})$. The reaction was stirred at that Temp for another $12 \mathrm{~h}$ before being diluted with DCM $(2 \mathrm{~mL})$ and brine $(2 \mathrm{~mL})$. The mixture was warmed up to $\mathrm{rt}$ and the inorganic phase was extracted with ethyl acetate $(3 \mathrm{X})$. The combined organic layers were dried over anhydrous magnesium sulfate and concentrated under pressure to give pale yellow residue. Column chromatography on silicon gel $(50 \%$ EtOAc/hexanes eluent) gave the desired product as pale yellow oil. (4.8mg, 48\%, single diastereomer, only in one case, recovered $29 \%$ starting material, in all other cases no 
starting material or other products were observed). ${ }^{1} \mathrm{H}$ NMR (400 MHz, CDCl3) $\delta 6.21$ $(\mathrm{dd}, \mathrm{J}=10.4,14.8 \mathrm{~Hz}, 1 \mathrm{H}), 5.87(\mathrm{~d}, \mathrm{~J}=10.0 \mathrm{~Hz}, 1 \mathrm{H}), 5.47(\mathrm{dd}, \mathrm{J}=8.4,14.8 \mathrm{~Hz}, 1 \mathrm{H})$, $4.00-3.92(\mathrm{~m}, 1 \mathrm{H}), 3.77-3.71(\mathrm{~m}, 1 \mathrm{H}), 3.64(\mathrm{~s}, 3 \mathrm{H}), 3.49(\mathrm{~s}, 3 \mathrm{H}), 3.29(\mathrm{~d}, \mathrm{~J}=10.0 \mathrm{~Hz}$, $1 \mathrm{H}), 3.06(\mathrm{t}, \mathrm{J}=4.8 \mathrm{~Hz}, 1 \mathrm{H}), 2.57(\mathrm{~d}, \mathrm{~J}=9.6 \mathrm{~Hz}, 1 \mathrm{H}), 2.56(\mathrm{dd}, \mathrm{J}=6.8,15.2 \mathrm{~Hz}, 1 \mathrm{H})$, 2.37 (dd, J = 6.8, 15.2 Hz, 1H), $2.45-2.33(\mathrm{~m}, 1 \mathrm{H}), 1.84-1.80(\mathrm{~m}, 1 \mathrm{H}), 1.78$ (dd, J = $5.2,14.0 \mathrm{~Hz}, 1 \mathrm{H}), 1.69(\mathrm{~s}, 3 \mathrm{H}), 1.69-1.64(\mathrm{~m}, 1 \mathrm{H}), 1.60-1.46(\mathrm{~m}, 2 \mathrm{H}), 1.36-1.15(\mathrm{~m}$, $3 \mathrm{H}), 1.26(\mathrm{~s}, 3 \mathrm{H}), 1.19(\mathrm{~d}, \mathrm{~J}=6.4 \mathrm{~Hz}, 3 \mathrm{H}), 1.03(\mathrm{~d}, \mathrm{~J}=6.4 \mathrm{~Hz}, 3 \mathrm{H}), 0.93(\mathrm{~d}, \mathrm{~J}=6.8 \mathrm{~Hz}$, $3 \mathrm{H}), 0.68(\mathrm{~d}, \mathrm{~J}=6.8 \mathrm{~Hz}, 3 \mathrm{H}) ;{ }^{13} \mathrm{C} \mathrm{NMR}(400 \mathrm{MHz}, \mathrm{CDCl} 3) \delta 171.8,139.1,135.2,127.6$, 125.0, 90.1, 86.7, 73.9, 68.2, 66.1, 61. 1, 60.3, 51.5, 46.8, 41.3, 34.9, 34.7, 32.4, 32.4, 31.6, 21.7, 18.9, 17.7, 16.6, 12.7, 11.9 ; IR (Neat) $v_{\max } 3443,2956,2925,2851,1740$, 1456, 1380, 1290, 1198, 1100. 1068, 968, 909; HRMS $\left(\mathrm{Cl} / \mathrm{NH}_{3}\right) \mathrm{m} / \mathrm{z}$ calcd for $\mathrm{C}_{26} \mathrm{H}_{44} \mathrm{O}_{6}$ $[\mathrm{M}+\mathrm{Na}]^{+} 475.3044$, found $475.3036 .[\alpha]_{20}{ }^{\mathrm{D}}=-21.7^{\circ}\left(c 0.5, \mathrm{CHCl}_{3}\right)$.

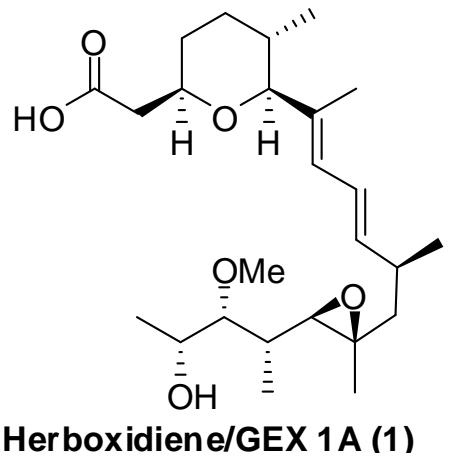

2-((2R,5S,6S)-6-((S,2E,4E)-7-((2R,3R)-3-((2R,3R,4R)4-hydroxy-3-methoxypentan-2-yl)-2-methyloxiran-2yl)-6-methylhepta-2,4-dien-2-yl)-5-methyltetrahydro2H-pyran-2-yl)acetic acid (1). A solution of $S$-alcohol $24(2.8 \mathrm{mg}, 6.2 \mu \mathrm{mol})$ in dry benzene $(0.01 \mathrm{M}, 0.5 \mathrm{~mL})$ was added $\mathrm{PPh}_{3} \quad(6.6 \mathrm{mg}, \quad 0.025 \mathrm{mmol})$ and $o$ chlorobenzoic acid $(3.5 \mathrm{mg}, 0.022 \mathrm{mmol})$ followed by slow addition of DIAD ( $5 \mu \mathrm{L}, 0.025 \mathrm{mmol})$. The yellow reaction mixture was stirred at $\mathrm{rt}$ for $12 \mathrm{~h}$ before the solvent was removed and gave a cloudy residue. Column chromatography by $30-50 \%$ EtOAc/hexanes gave the desired chlorobenzoic ester along with an inseparable impurity. (6.2mg in total)

A solution of the crude ester $(5.6 \mathrm{mg}, 9.5 \mu \mathrm{mol})$ in methanol $(0.02 \mathrm{M}, 0.55 \mathrm{~mL}))$ and water $27 \mu \mathrm{L}$ was added potassium carbonate $(8 \mathrm{mg}, 0.057 \mathrm{mmol})$. The reaction mixture was stirred at $40^{\circ} \mathrm{C}$ overnight before being cooled down to $\mathrm{rt}$. The clear reaction mixture was then diluted with EtOAc $(2 \mathrm{~mL})$ and water $(1 \mathrm{~mL})$. The inorganic phase was carefully acidified with $1 \mathrm{~N} \mathrm{HCl}$ till $\mathrm{PH}=4.0$. The inorganic phase was extracted with EtOAc (3X). The combined organic layers were washed with brine and concentrated under pressure to give a cloudy residue. Column chromatography on silicon gel by $50 \%$ EtOAc/hexanes removed impurities and 7\% MeOH/DCM eluent gave the natural product as a colorless oil (1.8mg, 66\% in 2 steps). ${ }^{1} \mathrm{H} \mathrm{NMR}\left(400 \mathrm{MHz}, \mathrm{CD}_{3} \mathrm{OD}_{3}\right) \delta 6.30$ (dd, J = 10.8, 14.8 Hz, 1H), $5.92(\mathrm{~d}, \mathrm{~J}=10.8 \mathrm{~Hz}, 1 \mathrm{H}), 5.50(\mathrm{dd}, \mathrm{J}=8.8,14.8 \mathrm{~Hz}, 1 \mathrm{H}), 3.81$ (quintet, $\mathrm{J}=6.4 \mathrm{~Hz}, 1 \mathrm{H}), 3.80-3.70(\mathrm{~m}, 1 \mathrm{H}), 3.54(\mathrm{~s}, 3 \mathrm{H}), 3.34(\mathrm{~d}, \mathrm{~J}=10.0 \mathrm{~Hz}, 1 \mathrm{H}), 2.98$ $(\mathrm{d}, \mathrm{J}=4.4,6.4 \mathrm{~Hz}, 1 \mathrm{H}), 2.65(\mathrm{~d}, \mathrm{~J}=9.2 \mathrm{~Hz}, 1 \mathrm{H}), 2.46(\mathrm{dd}, \mathrm{J}=7.2,15.6 \mathrm{~Hz}, 1 \mathrm{H}), 2.50-$ $2.38(\mathrm{~m}, 1 \mathrm{H}), 2.38$ (dd, J = 6.0, $15.6 \mathrm{~Hz}, 1 \mathrm{H}), 1.91(\mathrm{dd}, \mathrm{J}=4.4,13.2 \mathrm{~Hz}, 1 \mathrm{H}), 1.90-1.82$ $(\mathrm{m}, 1 \mathrm{H}), 1.70(\mathrm{~s}, 3 \mathrm{H}), 1.74-1.65(\mathrm{~m}, 1 \mathrm{H}), 1.60-1.43(\mathrm{~m}, 1 \mathrm{H}), 1.29(\mathrm{~s}, 3 \mathrm{H}), 1.40-1.22$ $(\mathrm{m}, 1 \mathrm{H}), 1.26-1.18(\mathrm{~m}, 1 \mathrm{H}), 1.11(\mathrm{~d}, \mathrm{~J}=6.4 \mathrm{~Hz}, 3 \mathrm{H}), 1.05(\mathrm{~d}, \mathrm{~J}=6.4 \mathrm{~Hz}, 3 \mathrm{H}), 0.84(\mathrm{~d}, \mathrm{~J}$ $=7.0 \mathrm{~Hz}, 3 \mathrm{H}), 0.70(\mathrm{~d}, \mathrm{~J}=6.8 \mathrm{~Hz}, 3 \mathrm{H}) ;{ }^{13} \mathrm{C} \mathrm{NMR}\left(400 \mathrm{MHz}, \mathrm{CDCl}_{3}\right) \delta 175.1,42.3,75.5$, $32.8,33.7,33.5,18.0,91.9,136.3,12.6,129.2$, 126.4, 140.5, 36.4, 22.4, 48.0, 62.7, 16.8, 67.8, 36.2, 11.5, 88.6, 69.9, 19.9, 61.9; IR (Neat) $v_{\max } 3470,2962,2926,2849,1724$, 1454, 1380, 1099, 1068; HRMS $\left(\mathrm{Cl} / \mathrm{NH}_{3}\right) \mathrm{m} / \mathrm{z}$ calcd for $\mathrm{C}_{25} \mathrm{H}_{42} \mathrm{O}_{6}[\mathrm{M}+\mathrm{Na}]^{+} 461.2879$, found $461.2881 ;[\alpha]_{20}{ }^{\mathrm{D}}=+4.9^{\circ}(\mathrm{c} 0.2, \mathrm{MeOH}) .{ }^{1} \mathrm{H}$ and ${ }^{13} \mathrm{C}$ NMR data for our synthetic herboxidiene/ GEX 1A (1) were compared with the natural product in Table 1 and Table 
2. The observed significant differences in the $\mathrm{C} 1-\mathrm{C} 3$ region were also reported in Kocieński's total synthesis (ref. 5a). This indicated that the data reported for natural herboxidiene was more likely from a carboxylate derivative rather than the free acid.

Table 1. ${ }^{1} \mathrm{H}$ NMR data for natural and synthetic herboxidiene/GEX 1A (1) ${ }^{3}$

\begin{tabular}{|c|c|c|c|c|c|c|}
\hline \multicolumn{4}{|c|}{ Natural herboxidiene/GEX 1A } & \multicolumn{3}{c|}{ Synthetic herboxidiene/GEX 1A } \\
\hline Position & $\delta$ & Multiplicity & $J / H z$ & $\delta$ & Multiplicity & $J / H z$ \\
\hline $\mathrm{H} 2_{\mathrm{A}}$ & 2.45 & $\mathrm{dd}$ & $14.1,6.6$ & 2.46 & $\mathrm{dd}$ & $15.6,7.2$ \\
\hline $\mathrm{H} 2_{\mathrm{B}}$ & 2.25 & $\mathrm{dd}$ & $14.1,7.5$ & 2.38 & $\mathrm{dd}$ & $15.6,6.0$ \\
\hline $\mathrm{H} 3$ & 3.76 & $\mathrm{~m}$ & - & $3.80-3.70$ & $\mathrm{~m}$ & - \\
\hline $\mathrm{H}_{\mathrm{A}}$ & $1.86-1.68$ & $\mathrm{~m}$ & - & $1.90-1.82$ & $\mathrm{~m}$ & - \\
\hline $\mathrm{H} 4_{\mathrm{B}}$ & 1.30 & $\mathrm{~m}$ & - & $1.40-1.22$ & $\mathrm{~m}$ & - \\
\hline $\mathrm{H} 5_{\mathrm{A}}$ & $1.86-1.68$ & $\mathrm{~m}$ & - & $1.74-1.65$ & $\mathrm{~m}$ & - \\
\hline $\mathrm{H} 5_{\mathrm{B}}$ & $1.26-1.12$ & $\mathrm{~m}$ & - & $1.40-1.22$ & $\mathrm{~m}$ & - \\
\hline $\mathrm{H} 6$ & 1.55 & $\mathrm{~m}$ & - & $1.60-1.43$ & $\mathrm{~m}$ & - \\
\hline $\mathrm{H} 6-\mathrm{Me}$ & 0.66 & $\mathrm{~d}$ & 6.6 & 0.70 & $\mathrm{~d}$ & 6.8 \\
\hline $\mathrm{H} 7$ & 3.34 & $\mathrm{~d}$ & 9.9 & 3.34 & $\mathrm{~d}$ & 10.0 \\
\hline $\mathrm{H} 8-\mathrm{Me}$ & 1.68 & $\mathrm{~s}$ & - & 1.70 & $\mathrm{~s}$ & - \\
\hline $\mathrm{H} 9$ & 5.90 & $\mathrm{~d}$ & 11.1 & 5.92 & $\mathrm{~d}$ & 10.8 \\
\hline $\mathrm{H} 10$ & 6.29 & $\mathrm{dd}$ & $15.0,10.8$ & 6.30 & $\mathrm{dd}$ & $14.8,10.8$ \\
\hline $\mathrm{H} 11$ & 5.45 & $\mathrm{dd}$ & $15.0,9.0$ & 5.50 & $\mathrm{dd}$ & $14.8,8.8$ \\
\hline $\mathrm{H} 12$ & 2.44 & $\mathrm{~m}$ & - & $2.50-2.38$ & $\mathrm{~m}$ & - \\
\hline $\mathrm{H} 12-\mathrm{Me}$ & 1.03 & $\mathrm{~d}$ & 6.6 & 1.05 & $\mathrm{~d}$ & 6.4 \\
\hline $\mathrm{H} 13_{\mathrm{A}}$ & 1.91 & $\mathrm{dd}$ & $13.1,4.3$ & 1.92 & $\mathrm{dd}$ & $13.2,4.4$ \\
\hline $\mathrm{H} 13_{\mathrm{B}}$ & $1.26-1.12$ & $\mathrm{~m}$ & - & $1.26-1.18$ & $\mathrm{~m}$ & - \\
\hline $\mathrm{C} 14-\mathrm{Me}$ & 1.27 & $\mathrm{~s}$ & - & 1.29 & $\mathrm{~s}$ & - \\
\hline $\mathrm{H} 15$ & 2.65 & $\mathrm{~d}$ & 9.6 & 2.65 & $\mathrm{~d}$ & 9.2 \\
\hline $\mathrm{H} 16$ & 1.45 & $\mathrm{~m}$ & - & $1.60-1.43$ & $\mathrm{~m}$ & - \\
\hline $\mathrm{C} 16-\mathrm{Me}$ & 0.83 & $\mathrm{~d}$ & 6.9 & 0.83 & $\mathrm{~d}$ & 7.0 \\
\hline $\mathrm{H} 17$ & 2.96 & $\mathrm{dd}$ & $6.0,4.5$ & 2.98 & $\mathrm{dd}$ & $6.4,4.4$ \\
\hline $\mathrm{H} 18$ & 3.78 & $\mathrm{dq}$ & $6.6,6.3$ & 3.81 & $\mathrm{quintet}$ & 6.4 \\
\hline $\mathrm{H} 19$ & 1.11 & $\mathrm{~d}$ & 6.6 & 1.11 & $\mathrm{~d}$ & 6.4 \\
\hline $\mathrm{OMe}$ & 3.52 & $\mathrm{~s}$ & - & 3.52 & $\mathrm{~s}$ & - \\
\hline
\end{tabular}

Table 2. ${ }^{13} \mathrm{C}$ NMR data for natural and synthetic herboxidiene/GEX 1A (1)

\begin{tabular}{|c|c|c|c|c|c|c|c|}
\hline Position & Natural $\delta$ & Synthetic $\delta$ & $\Delta \delta$ & Position & Natural $\delta$ & Synthetic $\delta$ & $\Delta \delta$ \\
\hline C1 & 179.8 & 175.1 & -4.7 & C12 & 36.5 & 36.4 & -0.1 \\
\hline C2 & 46.4 & 42.3 & -4.1 & C12-Me & 22.7 & 22.5 & -0.2 \\
\hline C3 & 77.0 & 75.5 & -1.5 & C13 & 48.1 & 48.0 & -0.1 \\
\hline C4 & 33.1 & 32.8 & -0.3 & C14 & 62.6 & 62.7 & +0.1 \\
\hline C5 & 33.7 & 33.7 & 0.0 & C14-Me & 16.8 & 16.8 & 0.0 \\
\hline C6 & 33.5 & 33.5 & 0.0 & C15 & 67.8 & 67.8 & 0.0 \\
\hline C6-Me & 18.2 & 18.0 & -0.2 & C16 & 36.4 & 36.3 & -0.1 \\
\hline C7 & 92.2 & 91.9 & -0.3 & C16-Me & 11.7 & 11.2 & -0.5 \\
\hline C8 & 136.5 & 136.3 & -0.2 & C17 & 88.6 & 88.6 & 0.0 \\
\hline C8-Me & 12.1 & 12.6 & +0.5 & C18 & 69.8 & 69.9 & +0.1 \\
\hline C9 & 129.5 & 129.2 & -0.3 & C19 & 19.9 & 19.9 & 0.0 \\
\hline C10 & 126.6 & 126.4 & -0.2 & OMe & 61.9 & 61.9 & 0.0 \\
\hline C11 & 140.5 & 140.5 & 0.0 & & & & \\
\hline
\end{tabular}

\footnotetext{
${ }^{3} \mathrm{H}$ NMR and ${ }^{13} \mathrm{C}$ NMR data for natural heroxidiene/GEX 1A were taken from ref. 5a.
} 
Supporting information:

\section{Total synthesis of Herboxidiene/GEX 1A}

Yun Zhang and James S. Panek*

Photocopies of selected ${ }^{1} \mathrm{H}$ NMR and ${ }^{13} \mathrm{C}$ NMR spectra

All spectra are arranged in the order of their appearance in the manuscript 

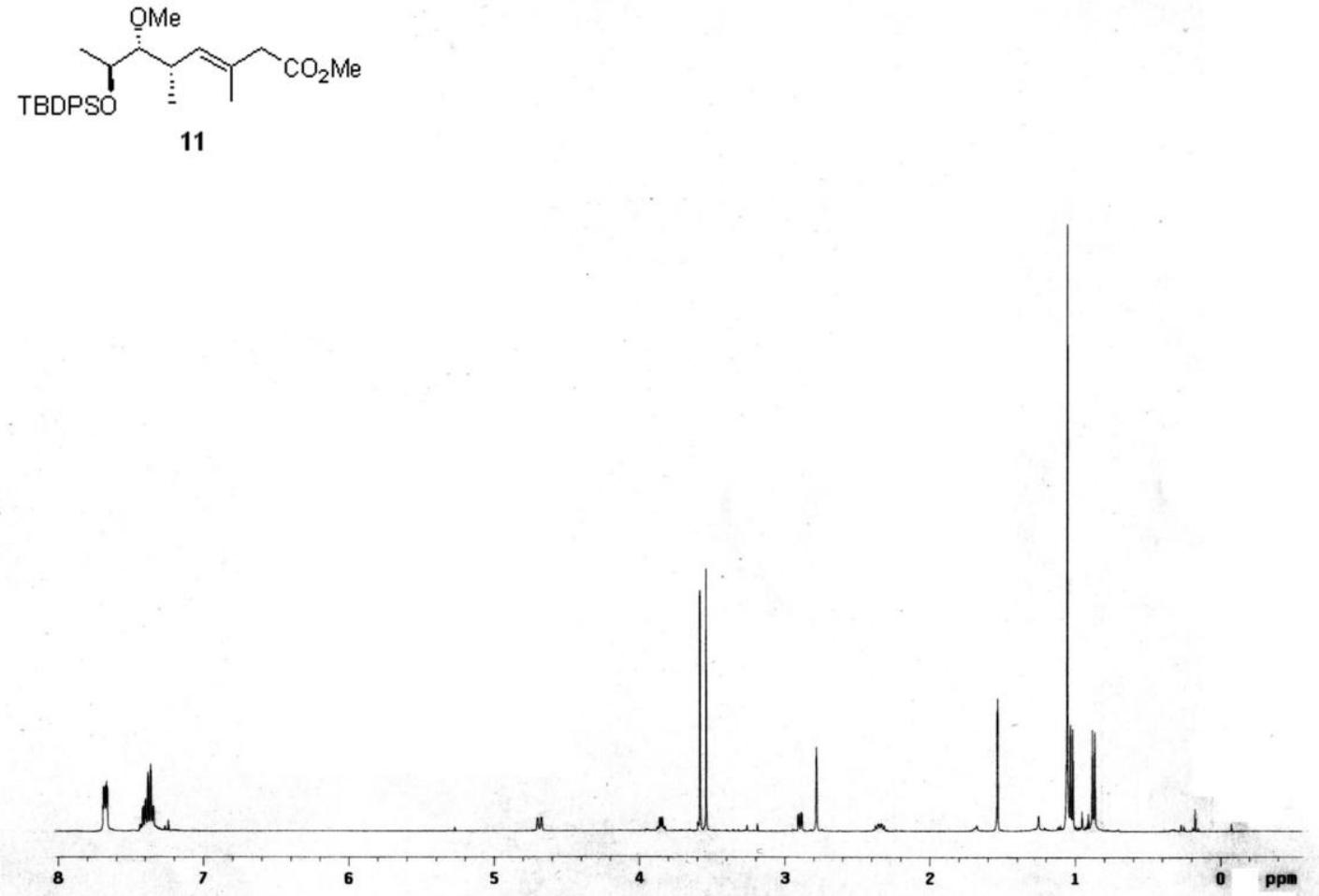

$\underbrace{\text { OMe }}_{\text {TBDPSO }}$

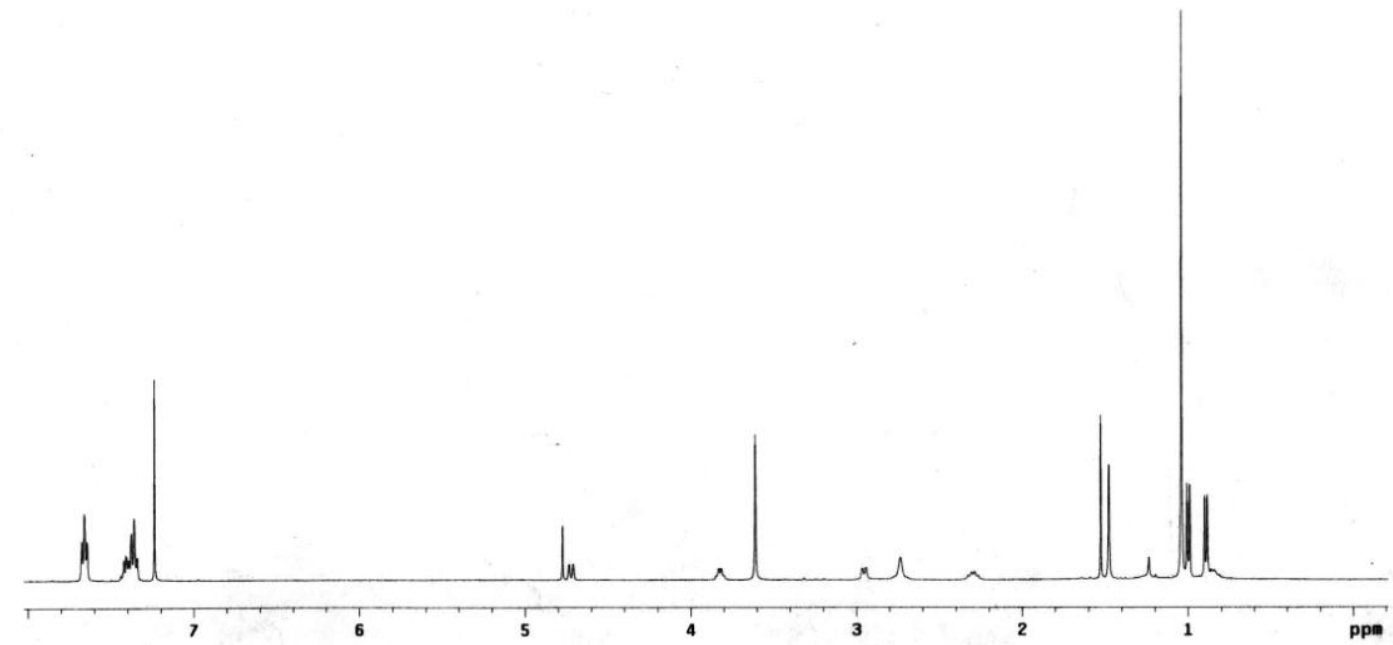



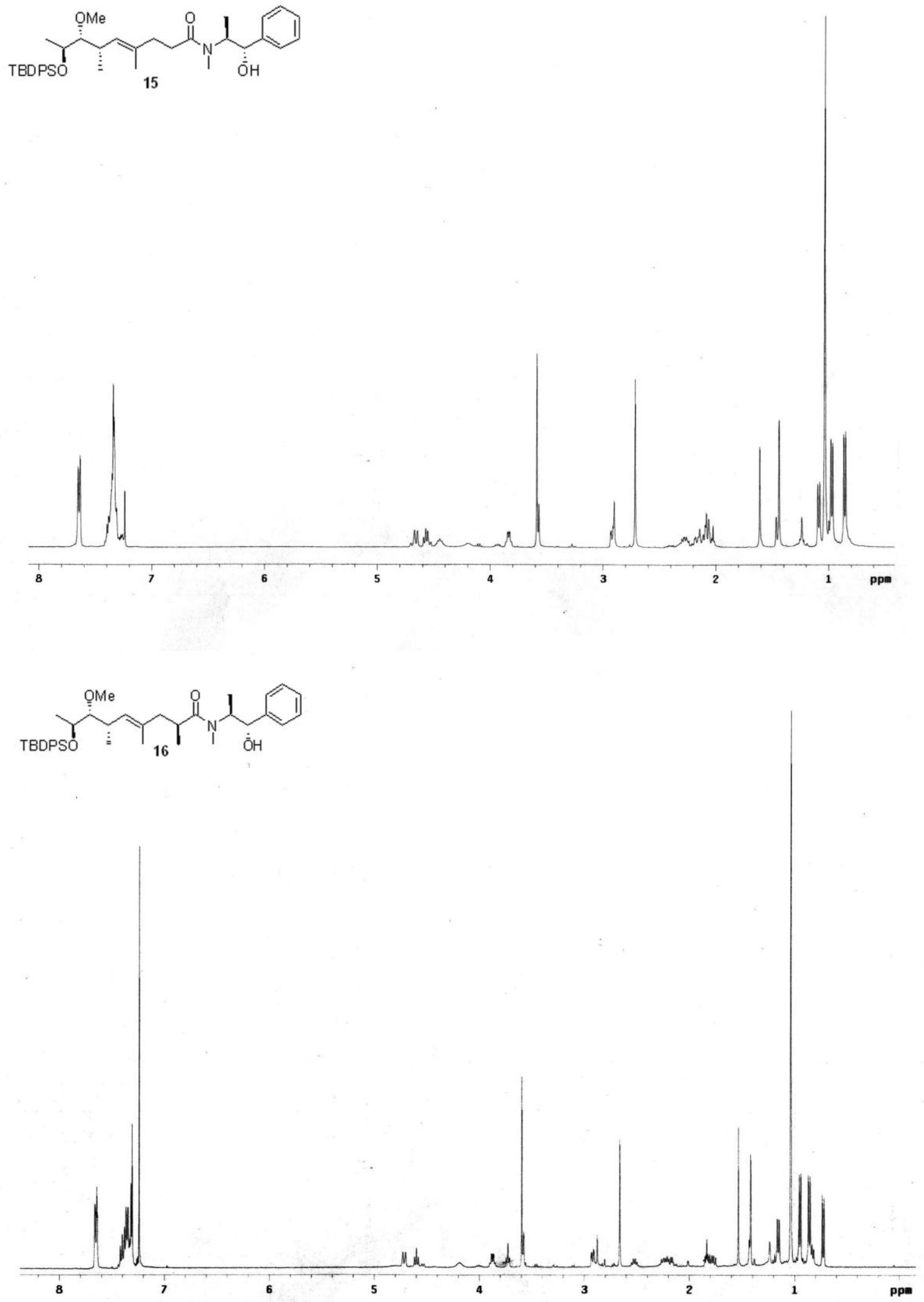

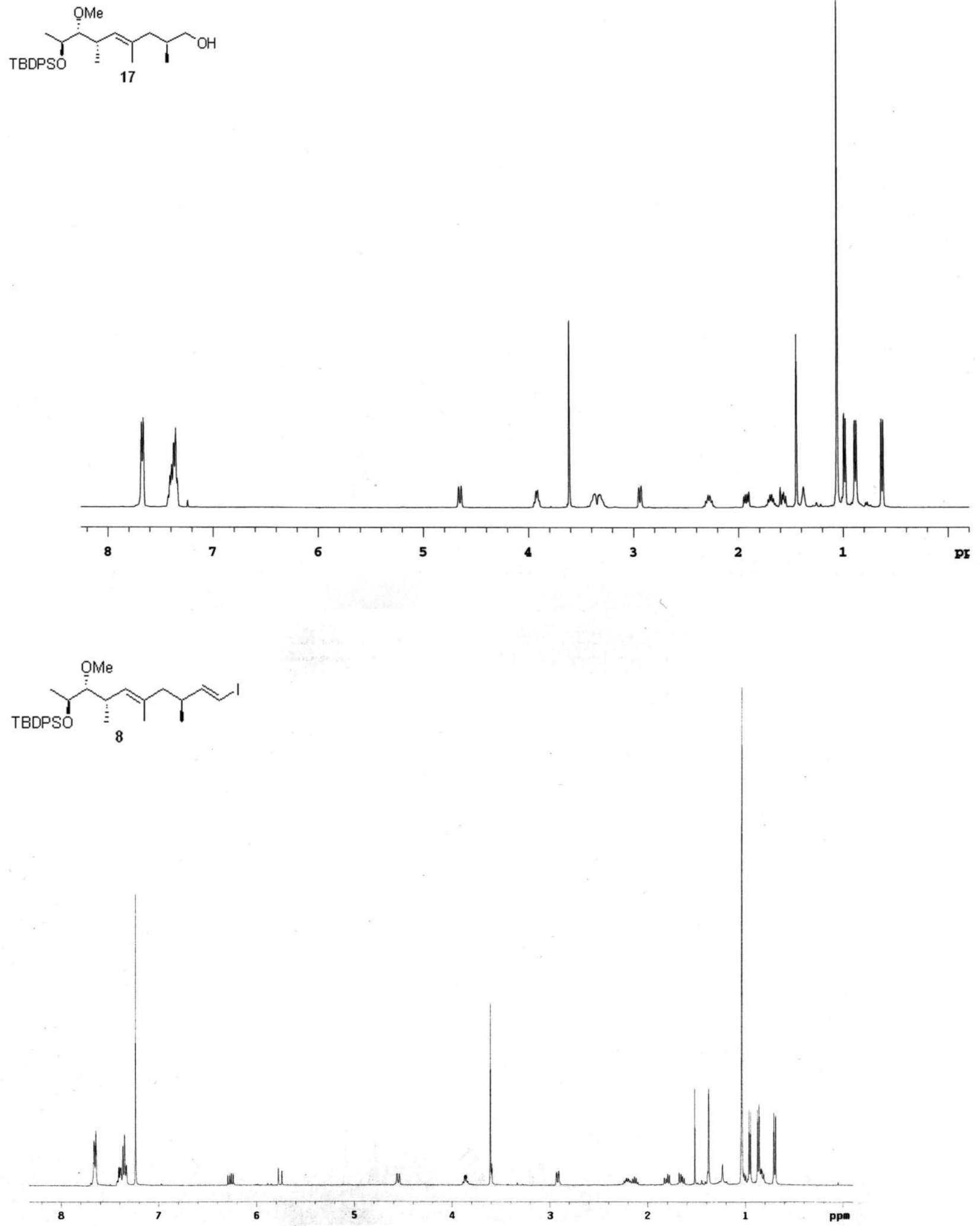

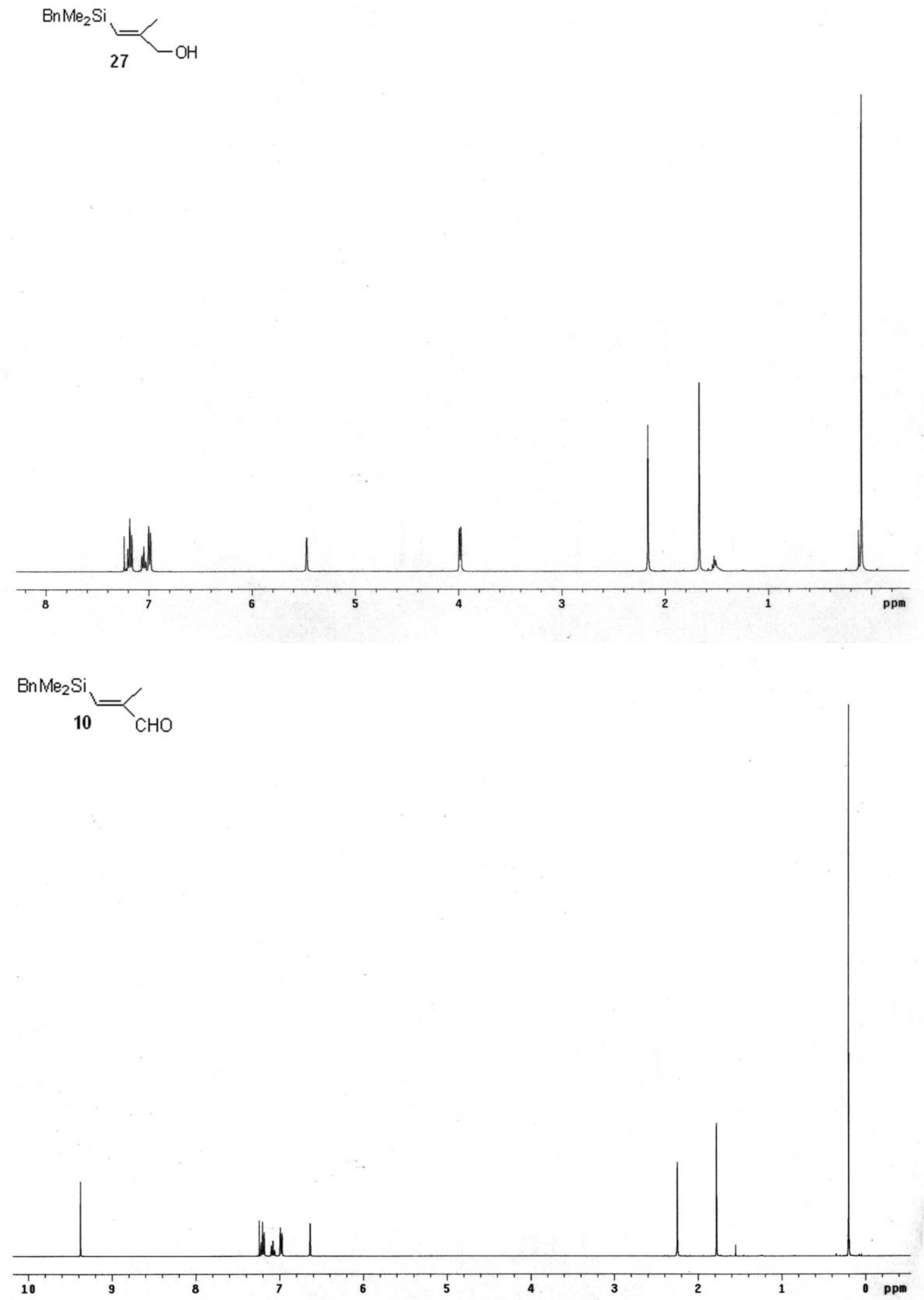

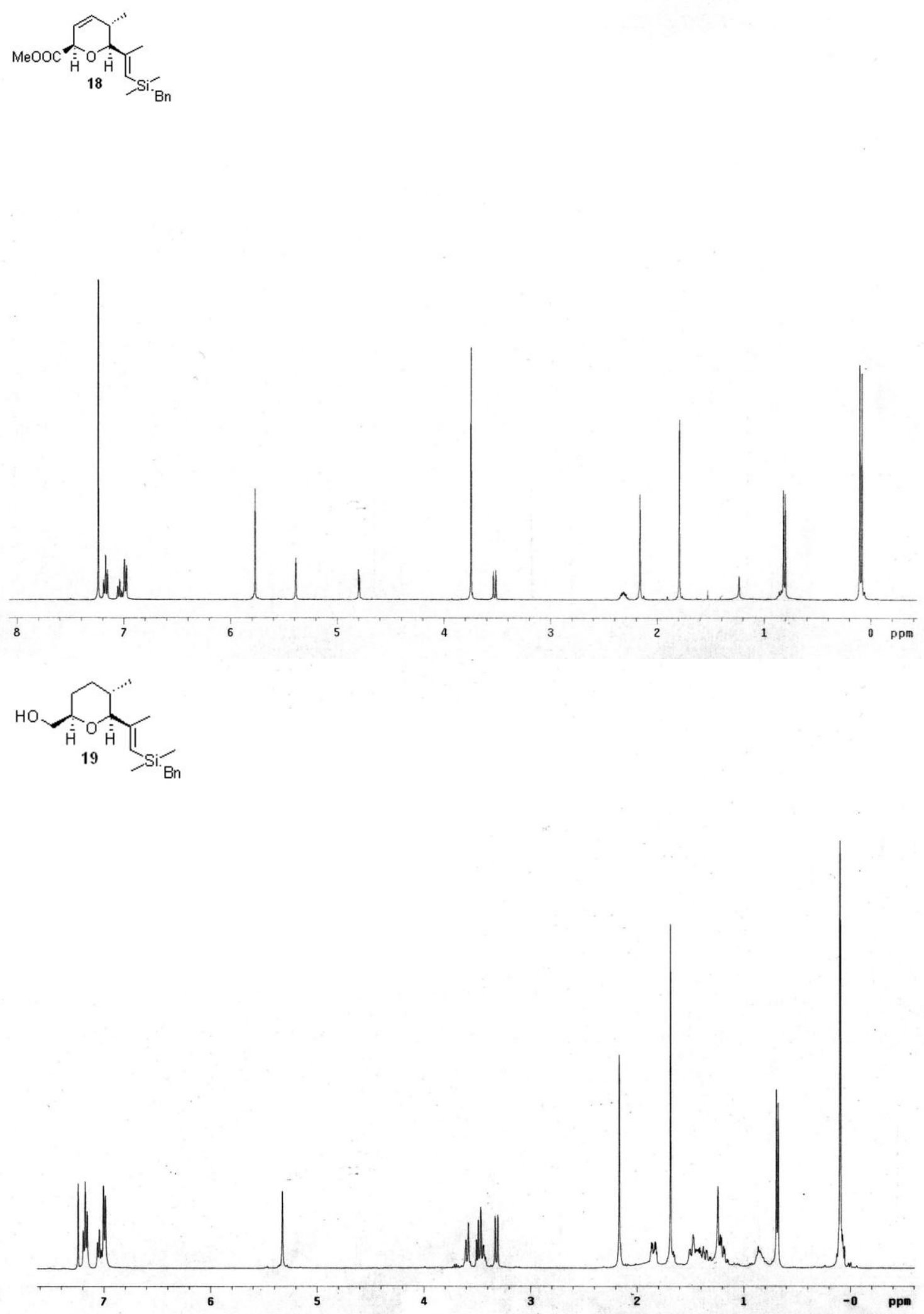

18 

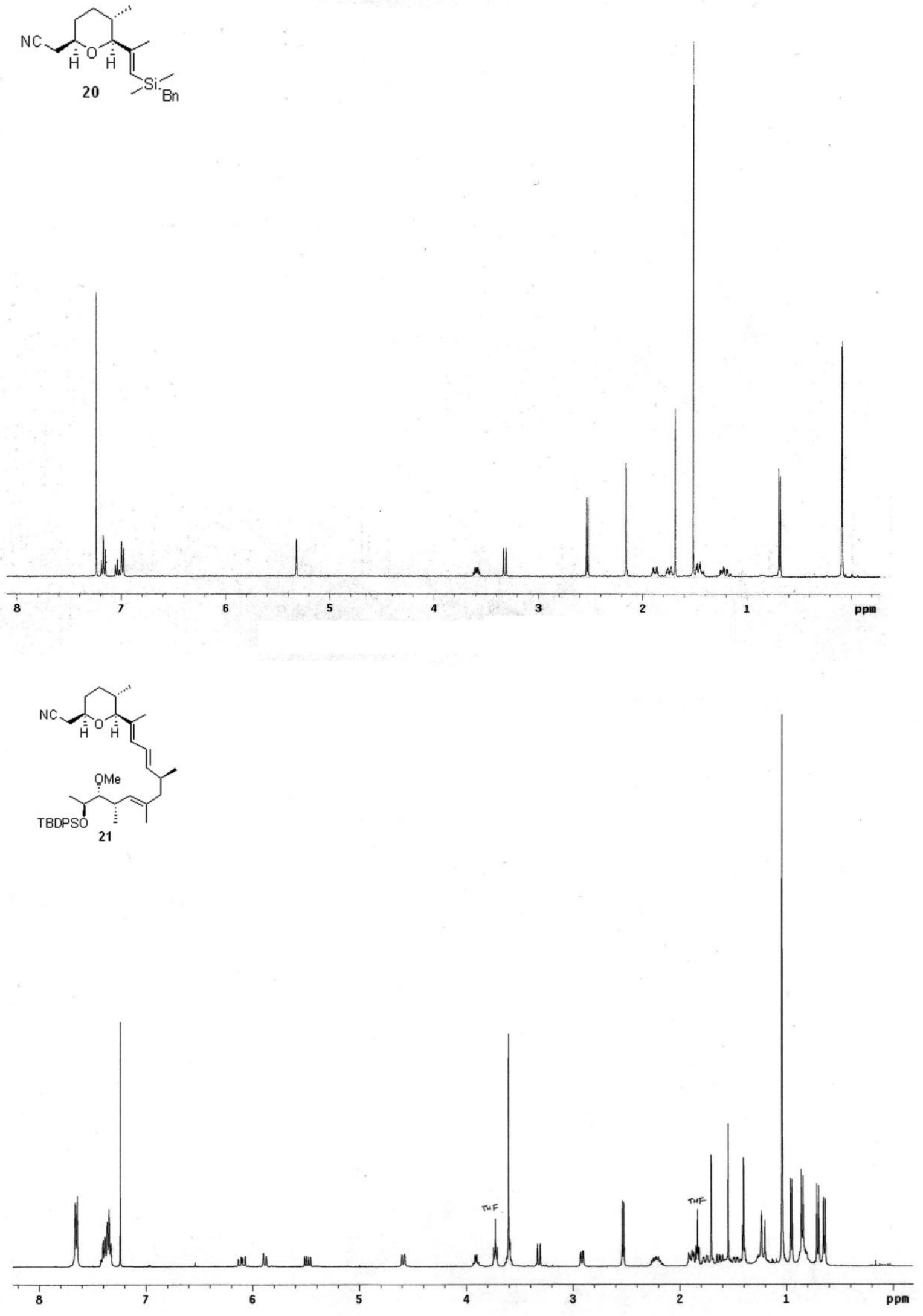

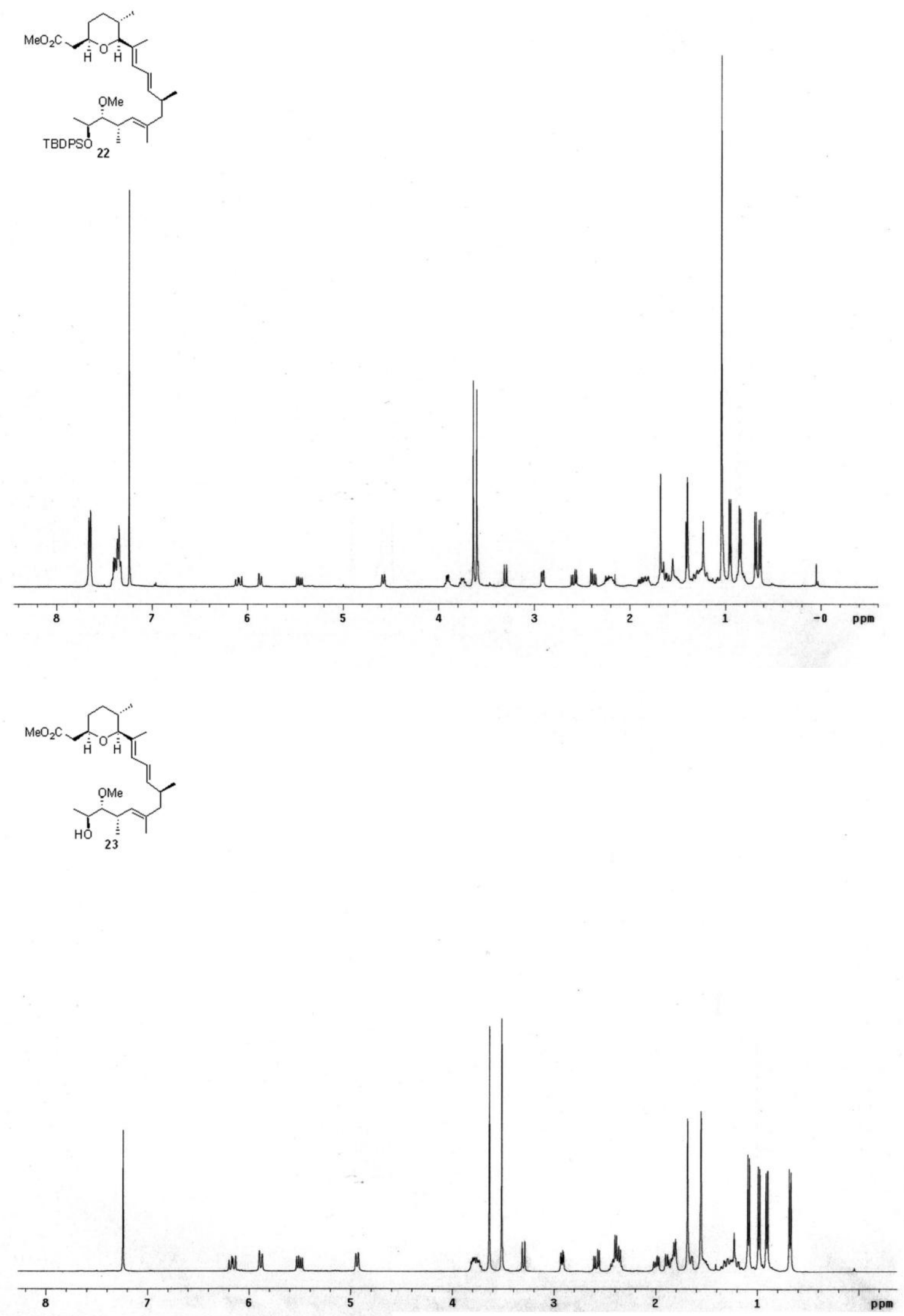

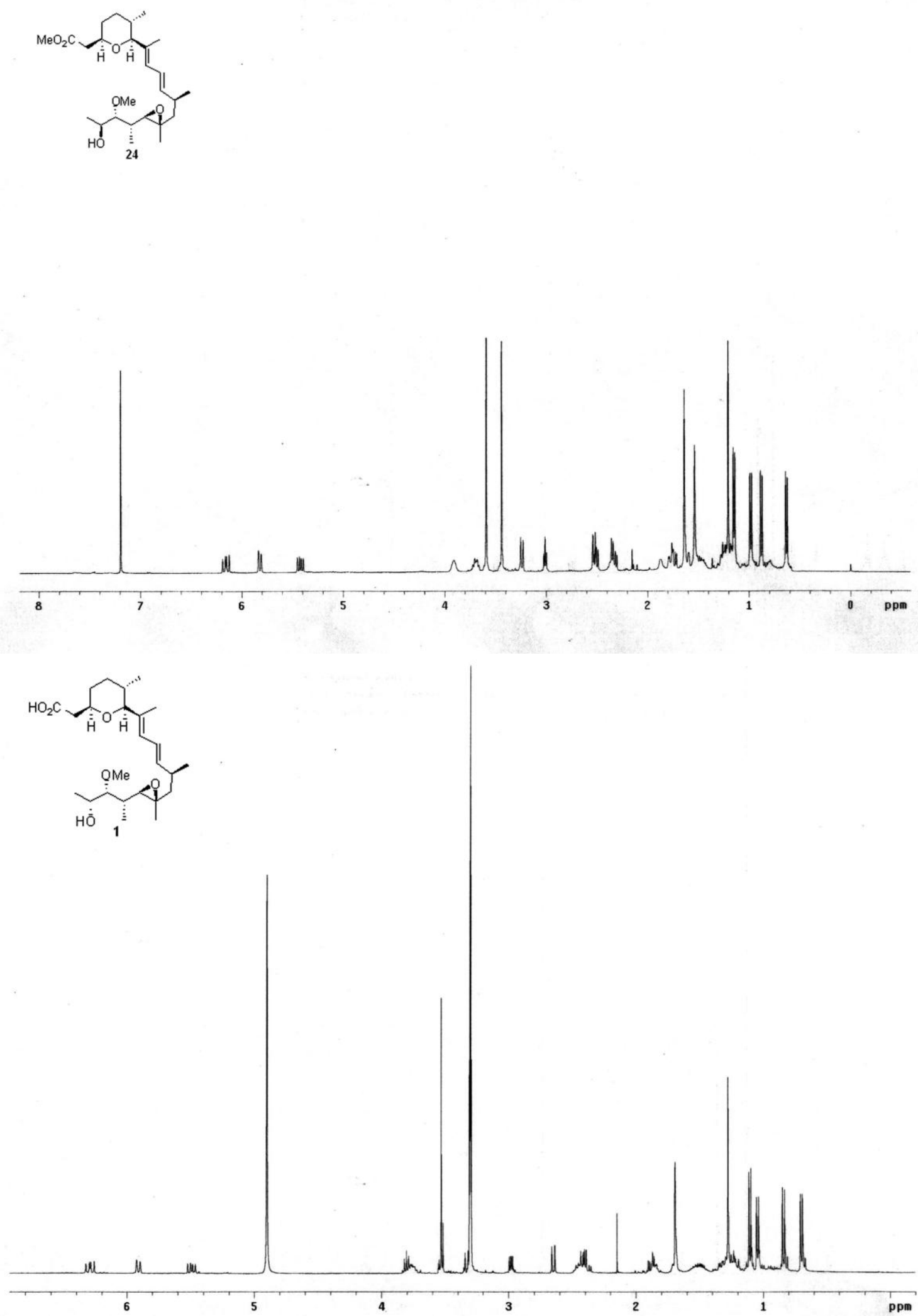

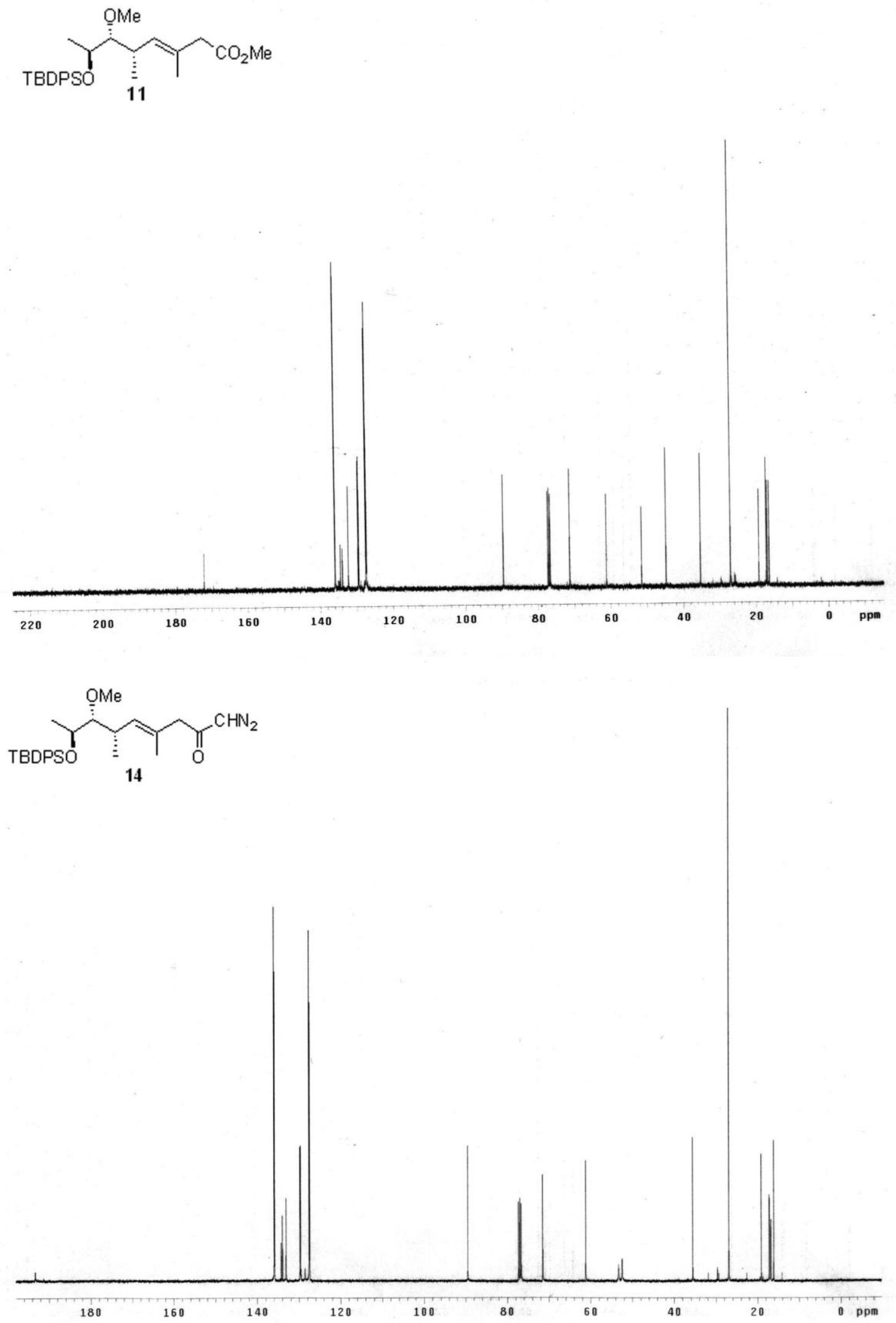

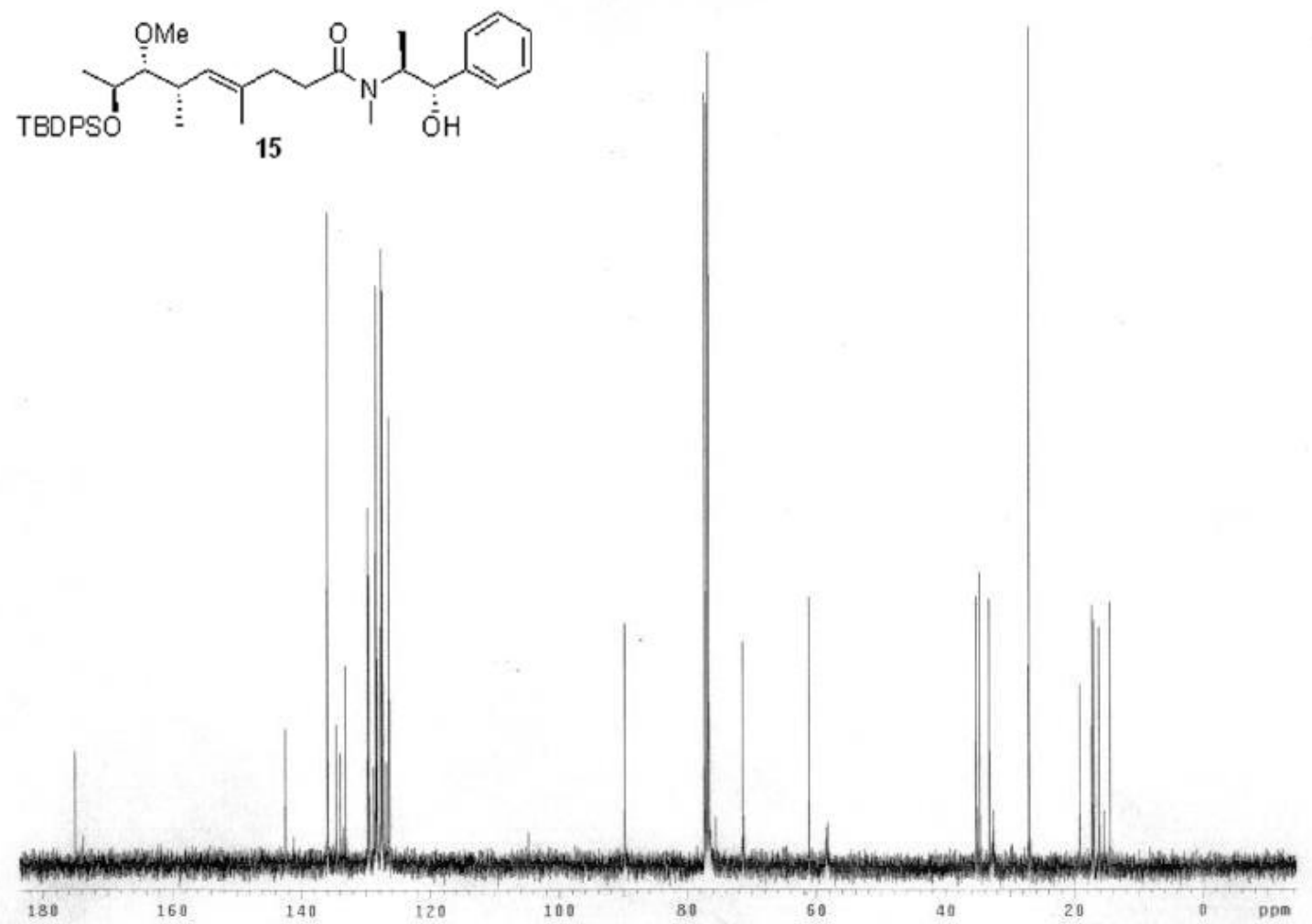<smiles>CCCCOC(C(C)OC)[C@@H](C)/C=C(\C)CC(C)C(=O)N(C)C(C)C(O)c1ccccc1</smiles>

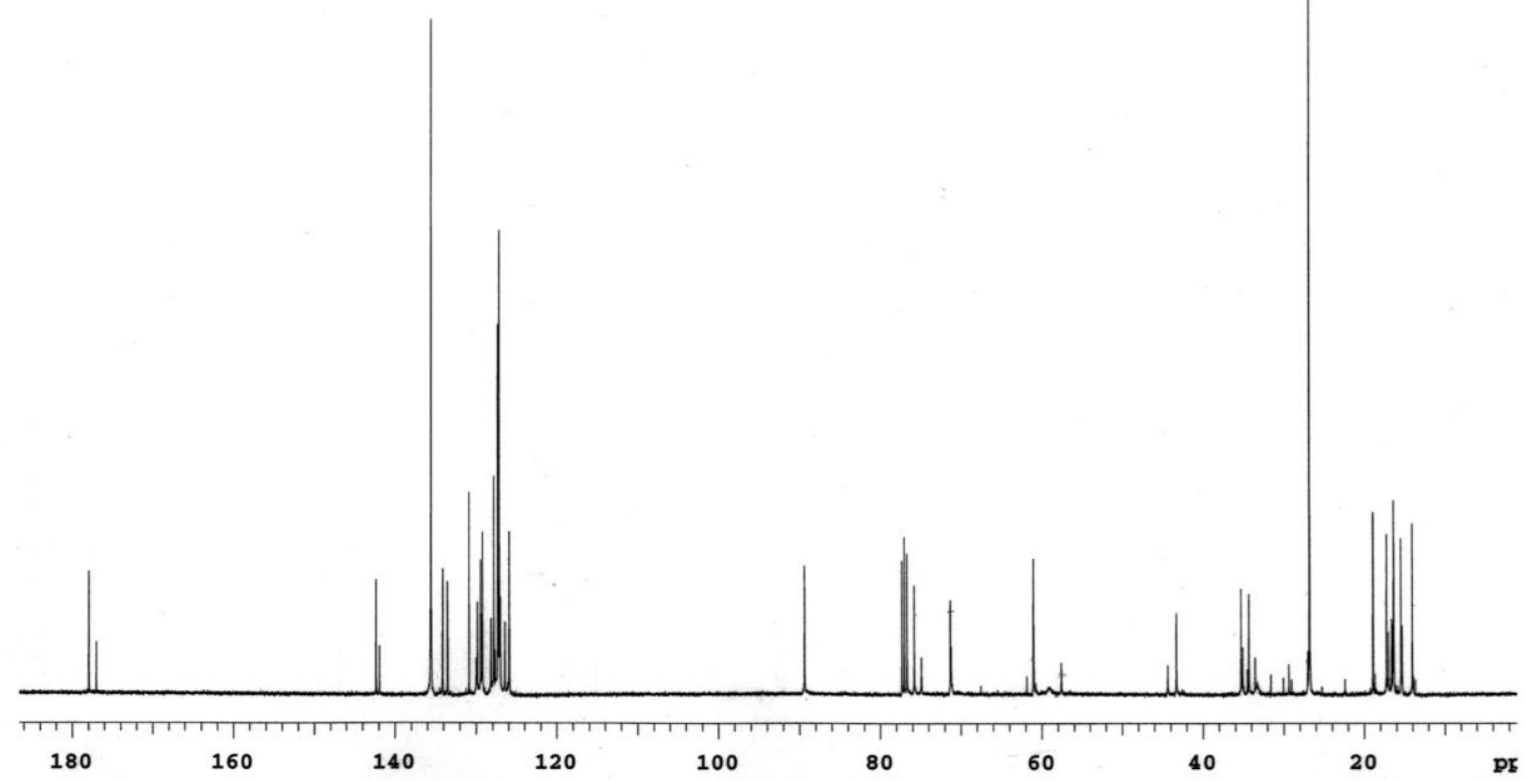



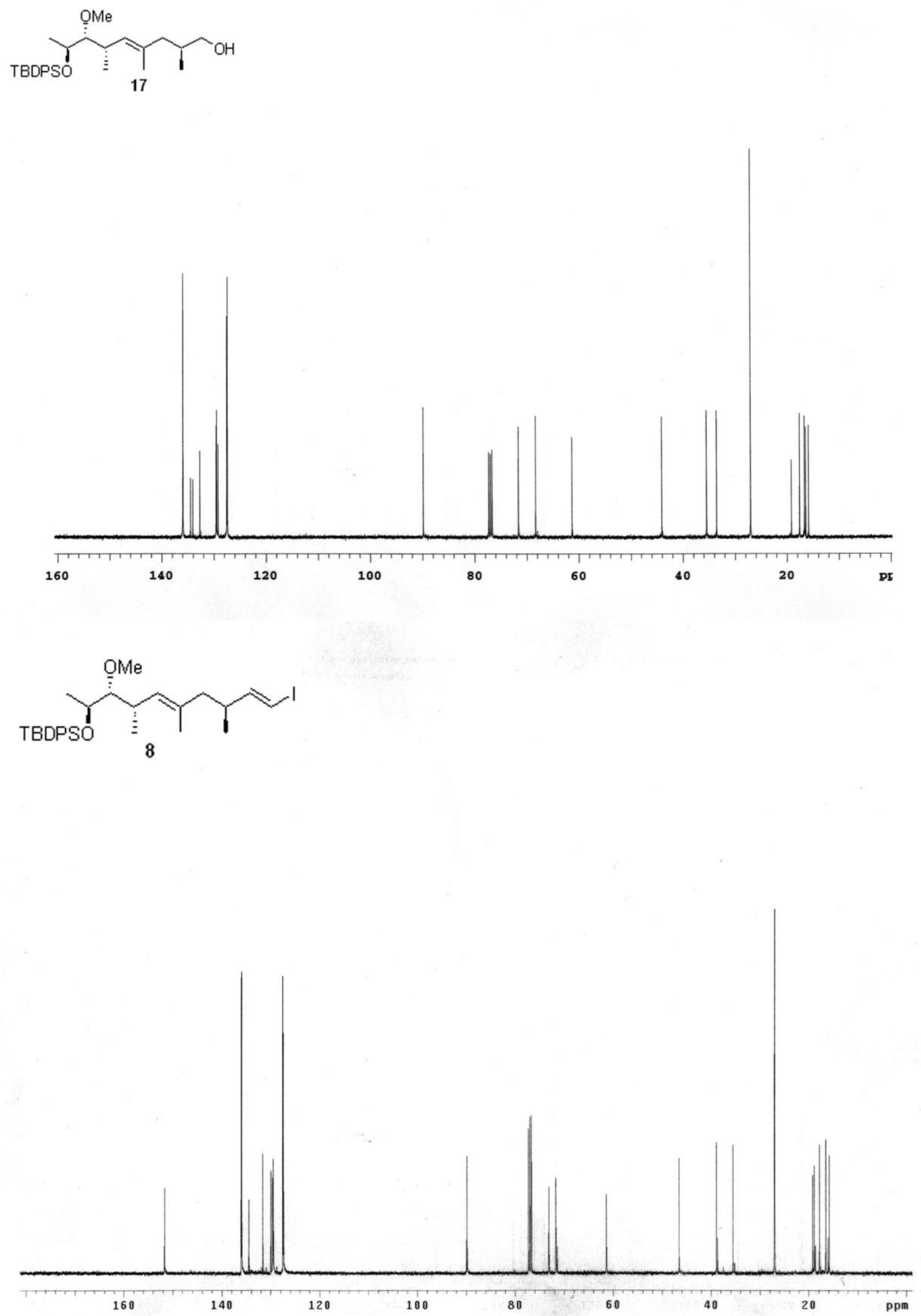

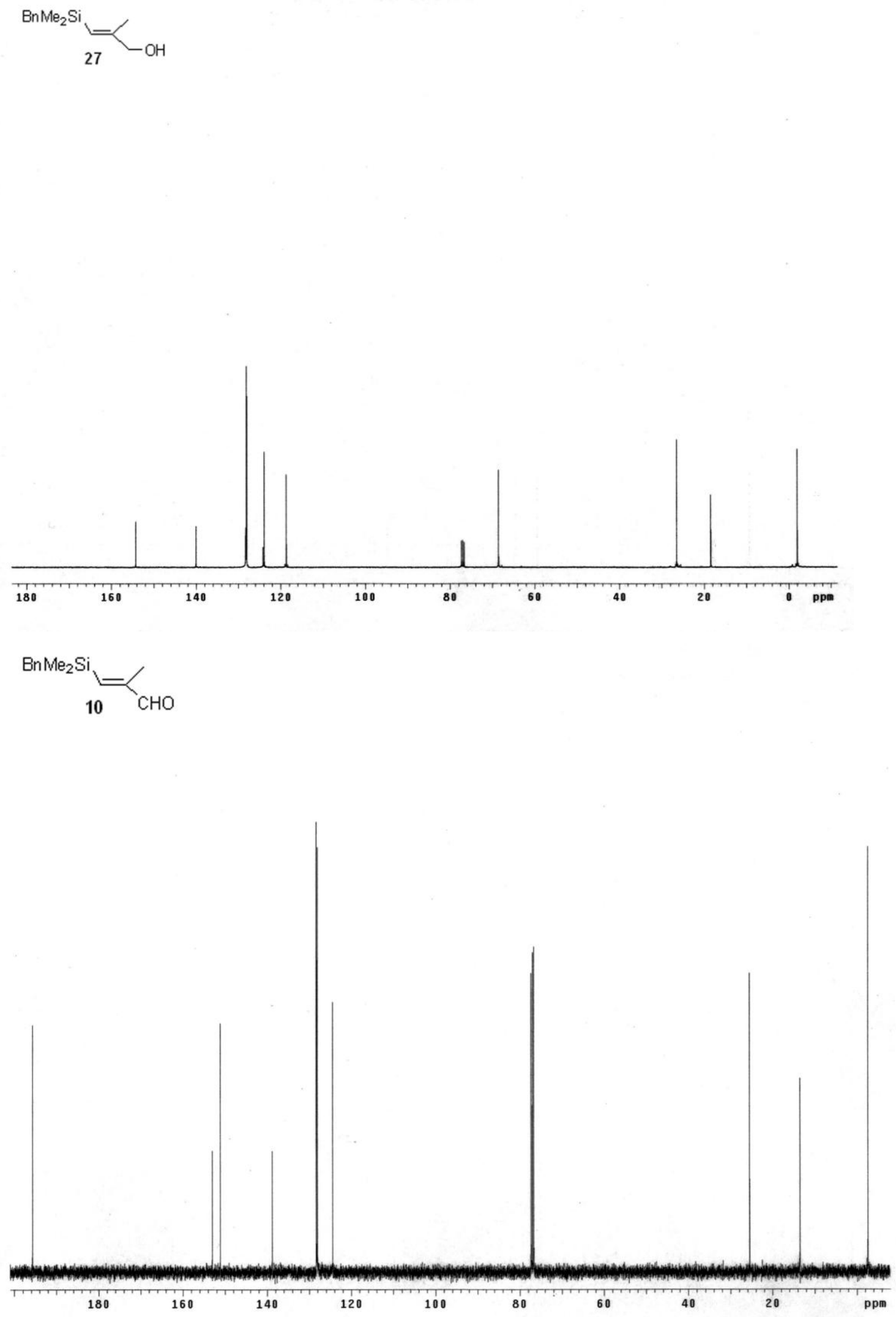

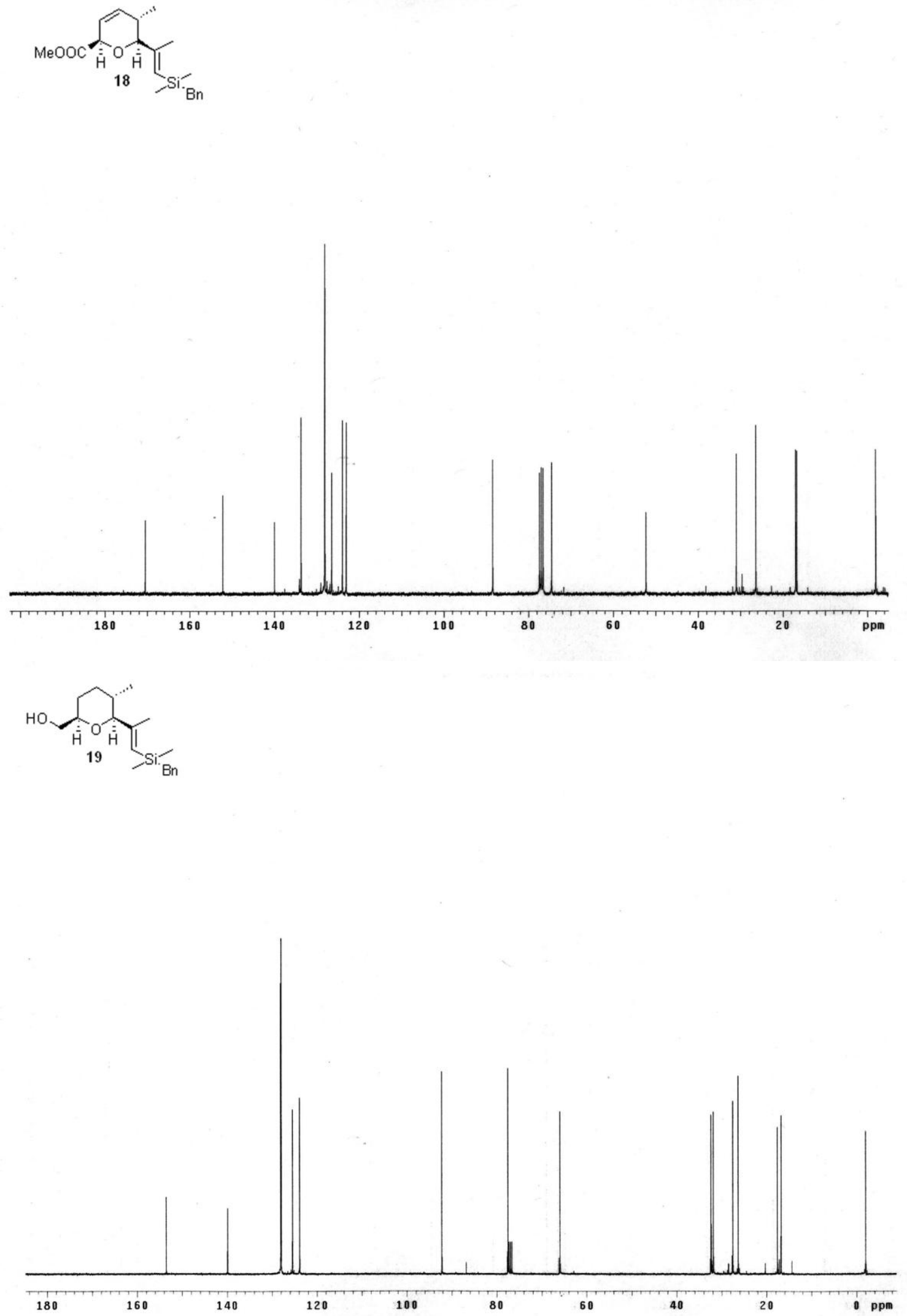

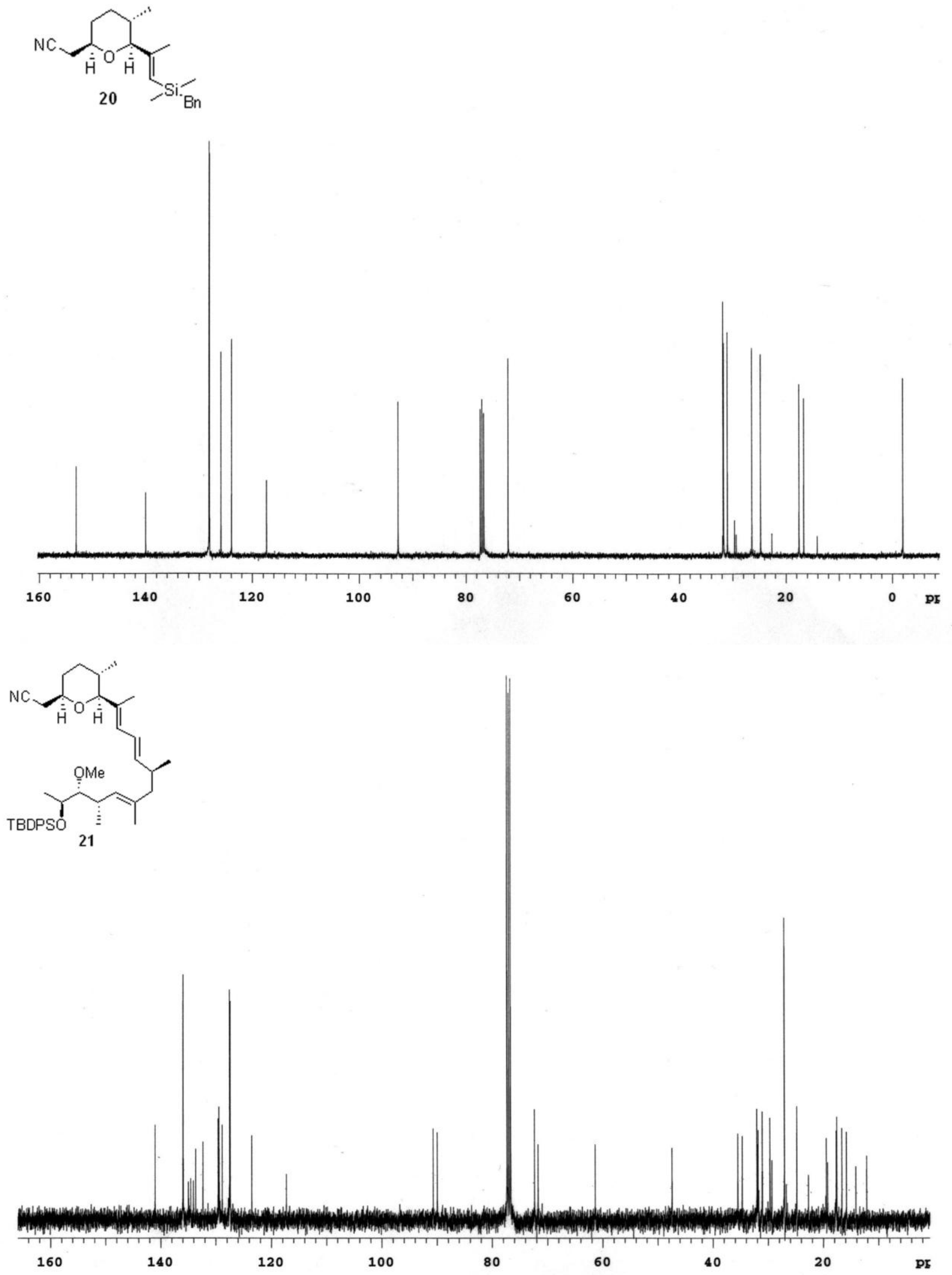

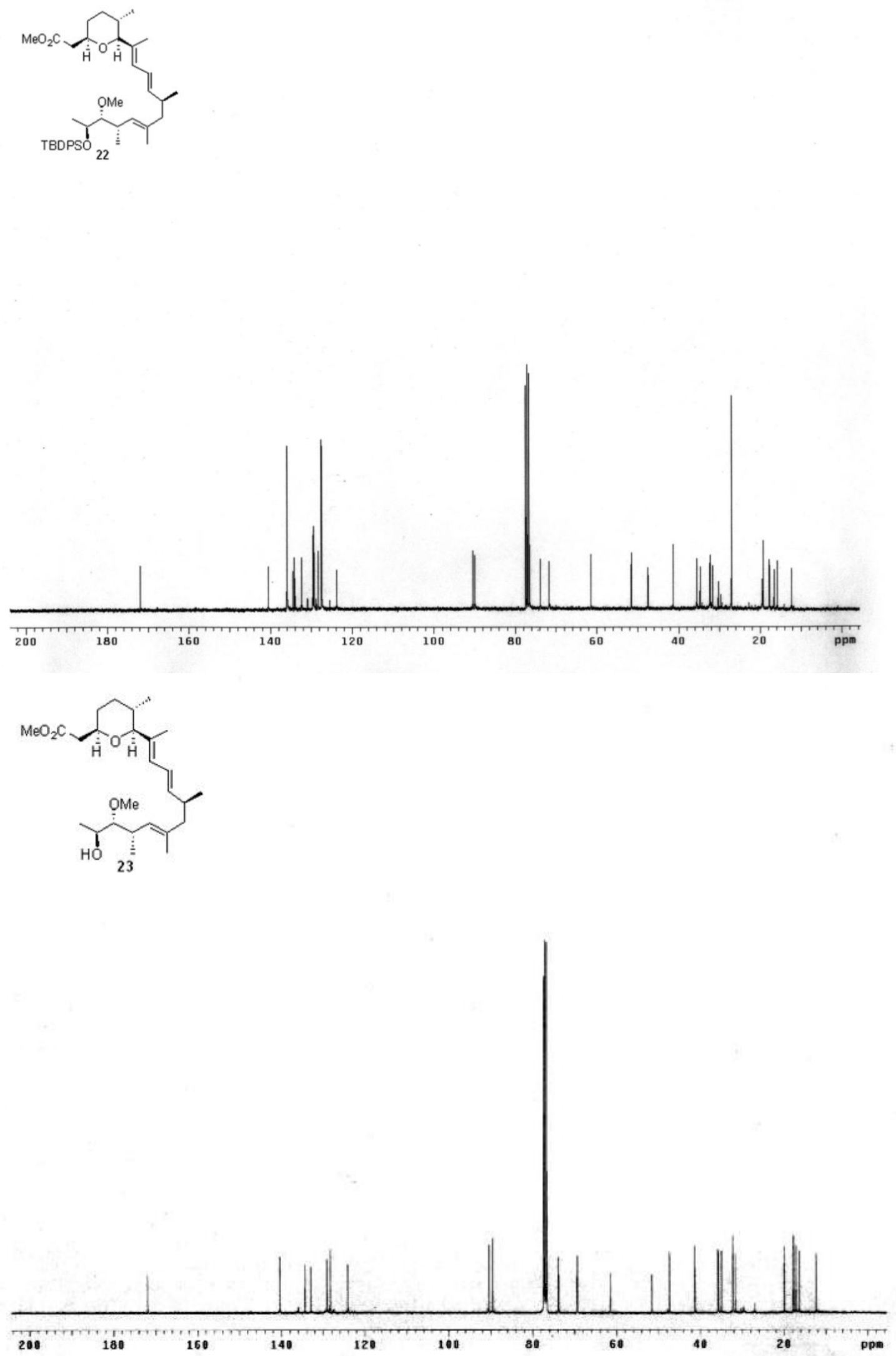

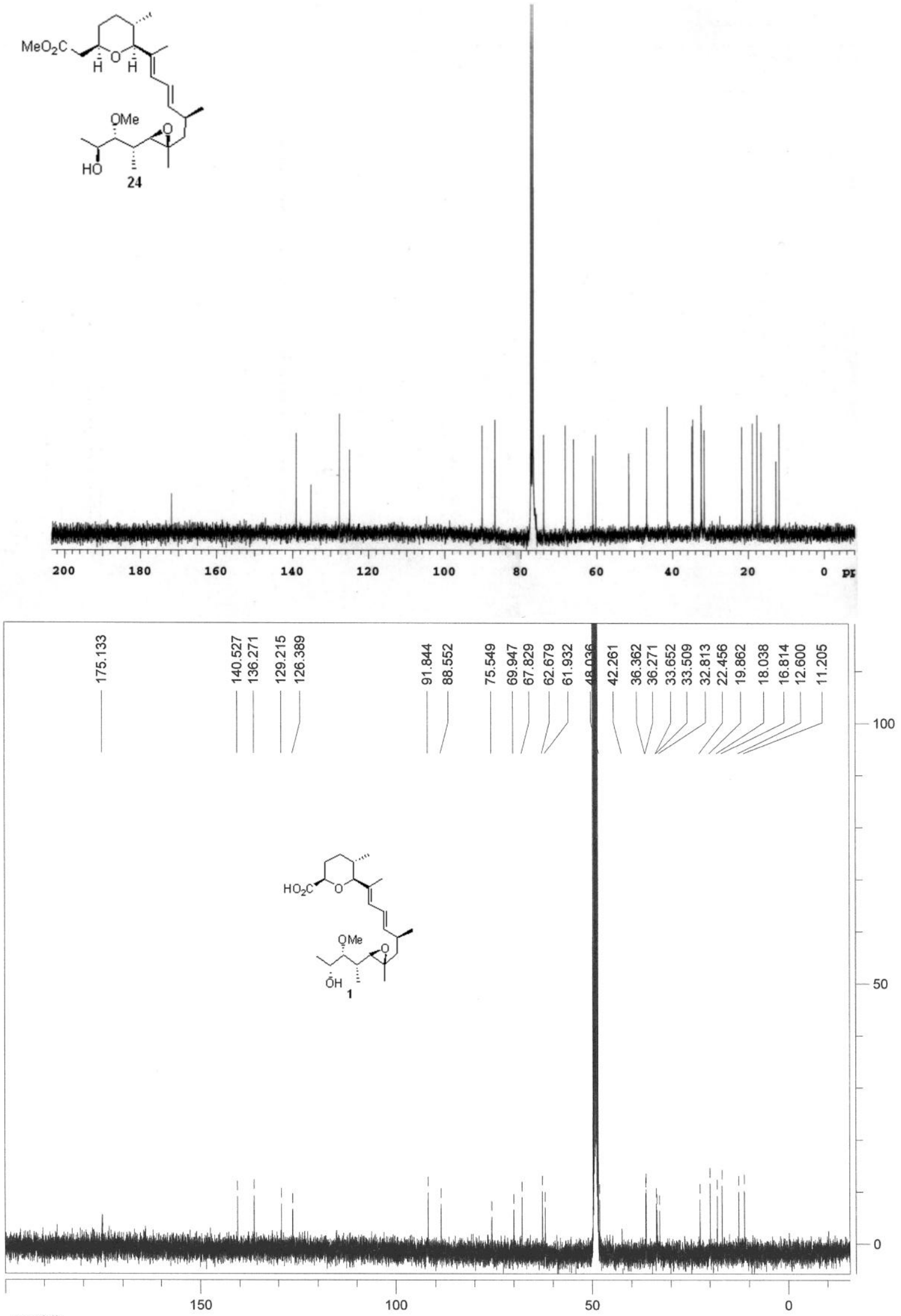